\title{
Tracking and Face Recognition of Multiple People Based on GMM, LKT and PCA
}

\author{
Won Oh Lee ${ }^{\dagger}$, Young Ho Park ${ }^{\dagger+}$, Eui Chul Lee ${ }^{+++}$, \\ HeeKyung Lee ${ }^{+++}$, Kang Ryoung Park ${ }^{++++}$
}

\begin{abstract}
In intelligent surveillance systems, it is required to robustly track multiple people. Most of the previous studies adopted a Gaussian mixture model (GMM) for discriminating the object from the background. However, it has a weakness that its performance is affected by illumination variations and shadow regions can be merged with the object. And when two foreground objects overlap, the GMM method cannot correctly discriminate the occluded regions. To overcome these problems, we propose a new method of tracking and identifying multiple people. The proposed research is novel in the following three ways compared to previous research: First, the illuminative variations and shadow regions are reduced by an illumination normalization based on the median and inverse filtering of the L*a*b* image. Second, the multiple occluded and overlapped people are tracked by combining the GMM in the still image and the Lucas-Kanade-Tomasi (LKT) method in successive images. Third, with the proposed human tracking and the existing face detection \& recognition methods, the tracked multiple people are successfully identified. The experimental results show that the proposed method could track and recognize multiple people with accuracy.
\end{abstract}

Key words: Intelligent surveillance system, tracking multiple people, GMM, LKT, face recognition

※ Corresponding Author: Kang Ryoung Park, Address: (100-715) Division of Electronics and Electrical Engineering, Dongguk University, Pil-dong 3-ga, Jung-gu, Seoul, Republic of Korea, TEL : +82-10-3111-7022, FAX :+82-2-2277- 8735, E-mail : parkgr@dgu.edu

Receipt date: Sep. 23, 2011, Revision date: Dec. 25, 2011 Approval date: Jan. 27, 2012

${ }^{+}$Division of Electronics and Electrical Engineering, Dongguk University, Seoul, Republic of Korea (E-mail: 215p8@hanmail.net)

${ }^{++}$Division of Electronics and Electrical Engineering, Dongguk University, Seoul, Republic of Korea (E-mail: fdsarew@hanafos.com)

${ }^{+++}$Department of Computer Science, Sangmyung University, Seoul, Republic of Korea (E-mail: oryong@hanmail.net)

${ }^{++++}$Broadcasting and Telecommunications Convergence Research Laboratory, Electronics and Telecommunications Research Institute (ETRI), Daejeon, Republic of Korea (E-mail: lhk95@etri.re.kr)

${ }^{+++++}$Division of Electronics and Electrical Engineering, Dongguk University, Seoul, Republic of Korea

* This work was supported by the R\&D program of KCC. (09912-03002, Development of Interactive View Control Technologies for IPTV).

\section{INTRODUCTION}

With the widespread use of closed circuit TV (CCTV) cameras, intelligent surveillance systems have become a hot research topic in the computer vision field [1,2]. These systems have various applications, such as access monitoring in a specific area, crowd congestion analysis, detection of abnormal behaviors [3], traffic monitoring, human identification at a distance, etc [1].

Previous researches are divided into two categories: the hardware-based and the softwarebased methods. The multimodal sensor-based system is an example of the hardware based method. It detects and identifies the objects of interest by using pyroelectricity infrared (PIR) sensors, audio sensors, or Doppler sensors [4-6]. Most of the software-based methods adopt the use of digital image processing and computer vision [1,7-31], which have the advantages of using 
cheaper camera systems and the obtaining of more detailed information of the object than the hardware-based ones. The software-based methods start with motion detection. Motion detection usually involves environment modeling and motion segmentation [1]. For environment modeling, many algorithms have been introduced, including the temporal average of an image sequence [22,23], adaptive Gaussian estimation [24], and parameter estimation based on the pixel processes [25,26]. Motion segmentation includes background subtraction [9,27,32], temporal difference [28], optical flow [29-31] and Gaussian mixture modeling (GMM) [14-17,22]. With the recent technological development of intelligent surveillance systems, it is required to track multiple persons robustly, taking into account the changes in illumination and background. However, most of previous studies into environment modeling and motion segmentation use the information from a still image or that found in successive images. The motion segmentation based on GMM, which has been used in most cases, has a weakness in that its performance is affected by illumination variations and in that the shadow regions can be merged with the object [14-17,22]. In order to solve these problems, many methods using color, texture, and reflectance estimations have been proposed [18,19]. However, these methods are too slow to be used in a real-time surveillance environment. In addition, when two foreground objects overlap, the GMM method cannot discriminate the occluded regions [33].

In previous researches, SVM-based combining method based on Retinex filtering and histogram stretching [34], and fuzzy-based Retinex filtering [35] have been introduced, which are designed for face recognition instead of real-time surveillance system.

In order to overcome these problems, we propose a new method of tracking and identifying multiple people. The proposed research is novel in the following three ways compared to previous research:
First, the illuminative variations and shadow regions are reduced by an illumination normalization based on the median and inverse filtering of the $\mathrm{L} * \mathrm{a} * \mathrm{~b} *$ image. Second, the multiple occluded and overlapped people are tracked by combining the GMM in the still image and the Lucas-KanadeTomasi (LKT) method in successive images. Third, with the proposed human tracking and the existing face detection \& recognition methods, the tracked multiple people are successfully identified.

Although many face recognition systems have been studied [36-41], there are few researches into the adoption of face recognition technology in a surveillance environment [20,21]. However, these few studies performed face detection and recognition using simple backgrounds or an indoor environment. Alternatively, the proposed method can track multiple people with face information, in indoor and outdoor environments having a complex background. The rest of this paper is organized as follows: The proposed method is explained in Section 2. Experimental results and conclusions are shown in Section 3 and 4, respectively.

\section{The Proposed Method}

\subsection{The overview of the proposed method}

Fig. 1 shows an overview of the proposed method. First, a RGB color image is captured and transformed into a $\mathrm{L} * \mathrm{a} * \mathrm{~b} *$ color image in order to extract the lightness component (L) for the reduction of the illumination variation of the captured image, as shown in step (1) of Fig. 1. The L image is blurred by the median filter, as shown in step (2) of Fig. 1 and the inversion of the L image is used for the illumination normalization, as shown in step (3) of Fig. 1. The illumination variation is reduced by adding the original captured image to the inverted one. The regions of interest (ROIs) of the human objects are extracted using the GMM method in the gray image, as shown in step (4) of Fig. 1. A more accurate human area is deter- 
mined through the morphological operation and the component labeling, as shown in step (5) of Fig. 1.

The edge points of the object are found by using the LKT method, as shown in step (6) of Fig. 1. Through analyzing the correspondences between the edge points in the current and the previous frames by the LKT method, the multiple motion vectors can be calculated, as shown in steps (7) and (8) of Fig. 1. The human area is determined based on the motion vectors, as shown in step (9) of Fig. 1. Then, the information of motion vectors is combined with the detected human region by the GMM, as shown in step (10) of Fig. 1. From that, the genuine human areas are continuously $\mathrm{ex}^{-}$ tracted, as shown in step (11) of Fig. 1, even if multiple objects are overlapped. After that, the face area is detected by using the Adaboost face detector in the predetermined search region in the human area, as shown in step (12) of Fig. 1.

The detected face area is then normalized into a $32 \times 32$ pixel image. Finally, as shown in step (13) of Fig. 1, the extracted face is matched with the previously enrolled face template by using the PCA based face recognition method.

\subsection{The foreground segmentation based on the GMM in the gray image}

In order to discriminate the moving foreground objects from the image, the background needs to be determined. The GMM is a very popular method

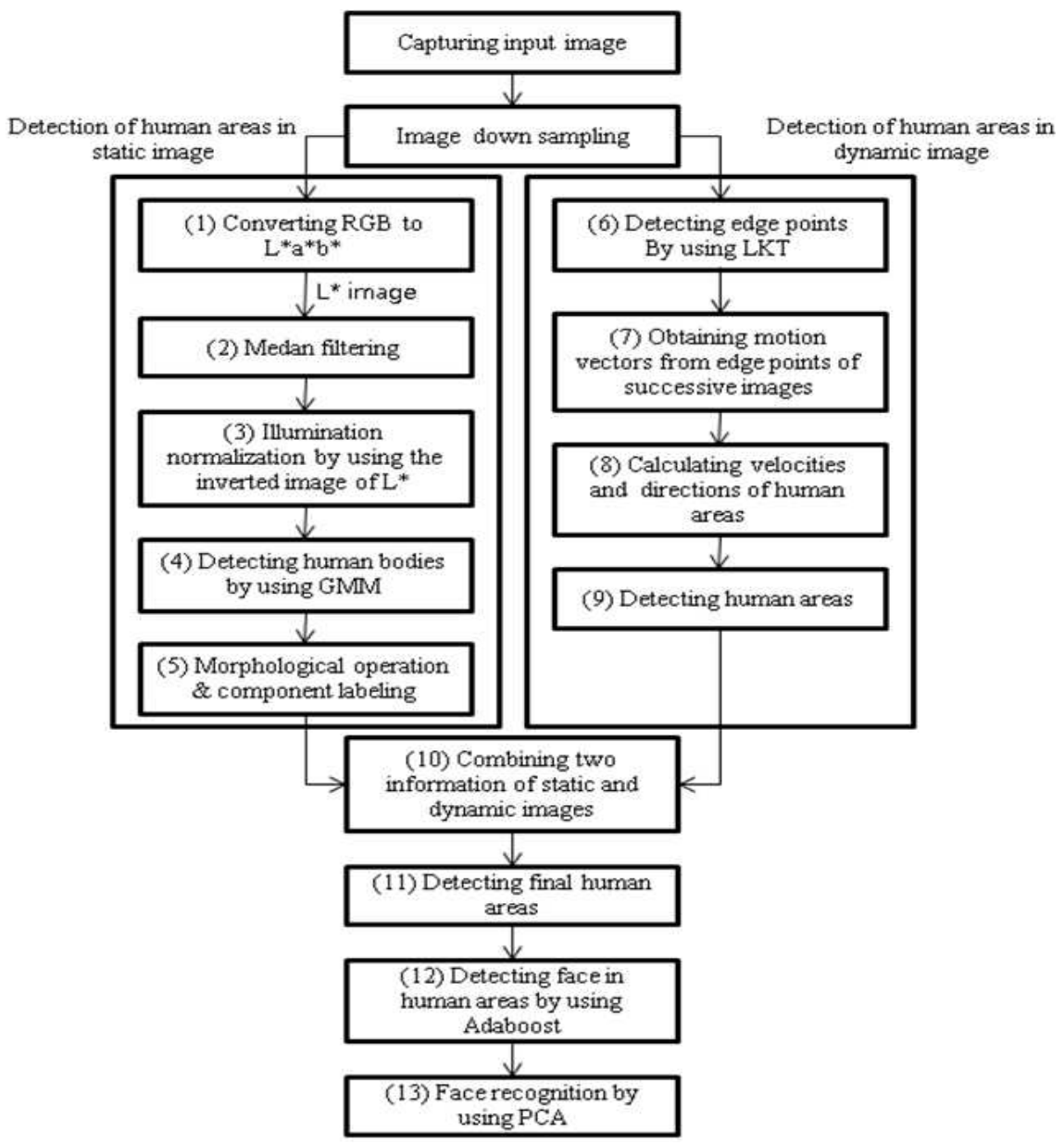

Fig. 1 A flow chart of the proposed method. 
used to segment the objects through the modeling background robust to a little amount of local change [32]. Assuming that the distributions of the pixel values define the corresponding probability density functions (PDF), the background and the foreground can be adaptively determined through the updating of the PDF and checking whether the input pixel value is included in the PDF of the background. This is when the Gaussian probability model is usually used to define the PDF. The Gaussian probability model is suitable to represent that the data is assembled to the center of the average. Although the Gaussian probability model is widely used, it is restricted to making a uni-modal distribution [42]. That is to say, the normal Gaussian model can represent only simple and slight changes of the pixel values. Therefore, we need a probability density function that is able to represent a more general form. This is the Gaussian mixture model and it is the sum of the plural number of the Gaussian probability model, and can be presented as the following equation [32,33]:

$$
P\left(X_{t}\right)=\sum_{i=1}^{K} \omega_{i, t} * \eta\left(X_{t}, \mu_{i, t}, \Sigma_{i, t}\right)
$$

where

$$
\eta\left(X_{t}, \mu, \Sigma\right)=\frac{1}{(2 \pi)^{\frac{n}{2}}|\Sigma|^{\frac{1}{2}}} e^{-\frac{1}{2}\left(X_{t}-\mu_{t}\right)^{T} \Sigma^{-1}\left(X_{t}-\mu_{t}\right)}
$$

And

$$
\omega_{i, t}=(1-\alpha) \omega_{i, t-1}+\alpha\left(M_{k, t}\right)
$$

where $a$ is the learning rate. $M_{k, t}$ is assigned as 1 for the model to be detected and 0 for that not to be detected. And $\mu$ is the mean, $\Sigma_{\mathrm{k}, \mathrm{t}}$ is the variance which is defined as $\Sigma_{k, t}=\sigma_{k}^{2} \mathbf{I}$. Here, $\mathbf{I}$ is unit matrix. After the Gaussians are ordered by the value of $\omega / a$, the first $B$ distributions are chosen as the background model.

$$
B=\operatorname{argmin}_{b}\left(\sum_{k=1}^{b} \omega_{k}>T\right)
$$

where $T$ is the minimum portion of the background model.

To increase the processing speed of the GMM, a 24-bit RGB color image, as shown in the left ones of Fig. 2, is converted to an 8-bit gray image, as shown in the middle ones of Fig. 2. Then, as shown in the right ones of Fig. 2, the human area is $1^{-}$ calized using the GMM.
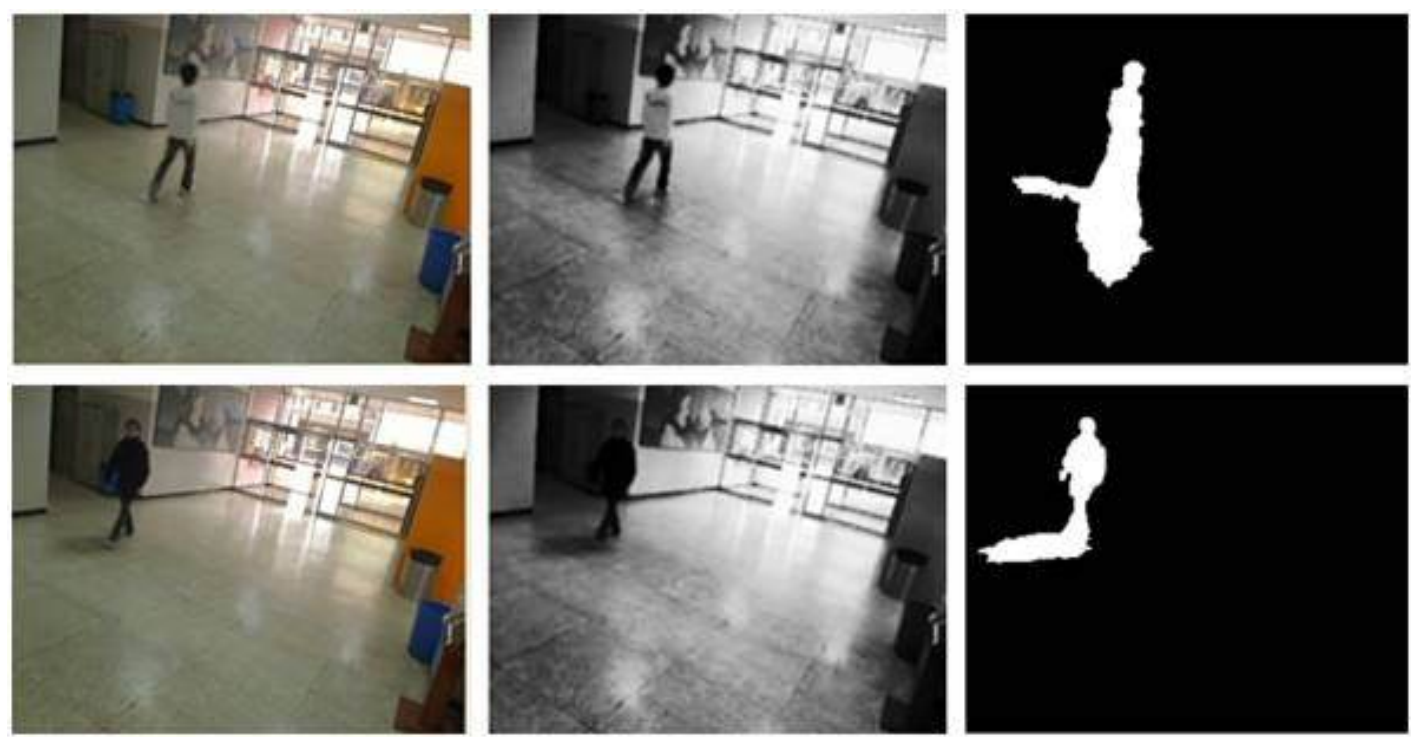

Fig. 2. The detection of the human area by the GMM. Left figures: the original image. Middle figures: the gray image. Right figures: the human area detected by using the GMM. 
The GMM based method has a problem in that it is sensitive to illuminative variations, as shown in the right figures of Fig. 3. To overcome this problem, we reduce the influence of the lightness by normalizing the illuminative component of the image before determining the human area.

\subsection{The illumination normalization method}

In general, the images captured by camera have the variation of environmental light or shadow as shown in Fig. 3.

Since there is no significant visual difference between areas (having the variation of light or shadow) and those without it in successive images, the correct foreground region is difficult to be discriminated only by the GMM. If the shadows or the light reflective areas are connected to a human area, the size of detected human area becomes bigger than the correct human area. If the shadows or light reflective areas are separate from the human area, there can be a problem that the separate areas are falsely recognized as being other human ones. Fig. 4 shows the examples that are influenced by light reflection or shadow areas.

In face recognition, illumination normalization methods based on Retinex filtering have been introduced, but they are too slow to be used in a real-time surveillance environment, since it uses time consuming Gaussian convolutions and logarithmic operations using the whole image [35]. So, a fast algorithm for solving this problem is proposed as follows:

After the RGB image is converted to the $\mathrm{L} * \mathrm{a} * \mathrm{~b} *$ image, an illuminative component image is acquired by applying the $5 \times 5$ pixel median filter to the $\mathrm{L}$ image, as shown in Fig. 5 (b). The L image includes all the factors of illumination variation, and the gray component of objects and background. Through median filtering, the illumination variation can be approximated excluding the gray component of objects and background as shown in Fig. 5 (b).
The inverted image is obtained from this, as shown in Fig. 5 (c). The influence of the illuminative variation is suppressed by summing the inverted image and the original $\mathrm{L}$ image, as shown in Fig. 5 (d). For example, the dark shadow on left bottom side of Fig. 5 (a) is reduced in Fig. 5 (d). In addition, the dark corridor (the left of a person) of Fig. 5 (a) becomes brighter in Fig. 5 (d). And the lighting of all parts of Fig. 5 (d) becomes more uniform than that of Fig. 5 (a).

The processing time of proposed illumination normalization is $8.6 \mathrm{~ms}$ in the input image of $320 \times 240$ pixels on average, while Retinex algorithm $[35,43]$ takes $96 \mathrm{~ms}$ in the image of same size. The total processing time is $90.6 \mathrm{~ms}$ (11 frames/sec) in a desktop computer, having an Intel® core 2TM Duo CPU 2.4GHz with 3GB RAM. Fig. 6 shows the results of the GMM based foreground extraction after applying the proposed illuminative normalization.

In previous research [44], the shadow of the foreground area can be removed on the basis that the color information of the shadow is similar to that of background in the detected foreground box, but the gray level of the shadow is different from that of background in the detected foreground box. However, in our experiments, the parts of foreground area (especially, a user wearing white clothes) were also incorrectly removed as the shadow by this method, but through the proposed illumination normalization, the shadows of foreground are removed while the most of foreground area are maintained.

Along with the detected foreground regions found by the illumination normalization and the GMM, additional post-processing methods, such as morphological operations and component labeling, are performed. In morphological operation, erosion and dilation are performed, through which some holes or concave regions can be filled and the uneven boundaries of foreground can be smooth. Through component labeling, the noise region whose size is small and isolated can be 

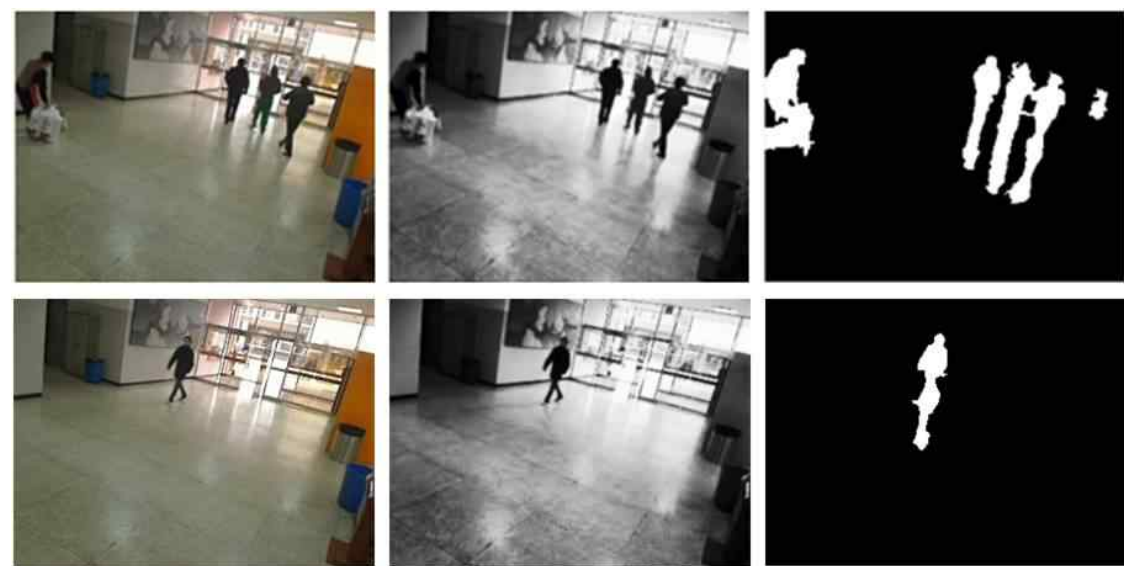

Fig. 3. The images having variations caused by light or shadow. Left figures: the original image. Middle figures: the gray image. Right figures: the segmented human areas having the reflection of the light or shadow.
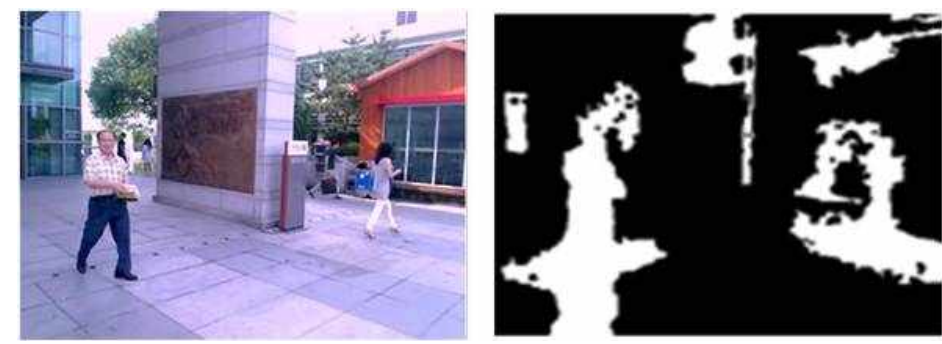

(a)
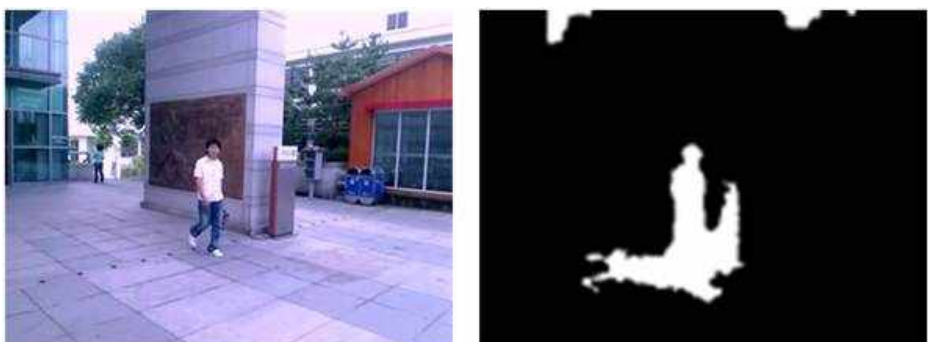

(b)
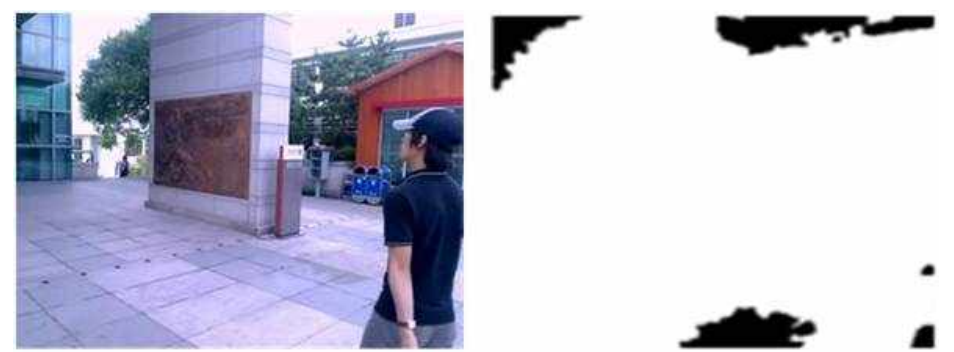

(c)

Fig. 4. Examples of images that include illuminative variation (left) and the resultant images using GMM (right). (a) the case of noise that is generated by illuminative variation. (b) the case including shadow. (c) the case including an abrupt change of illumination. 


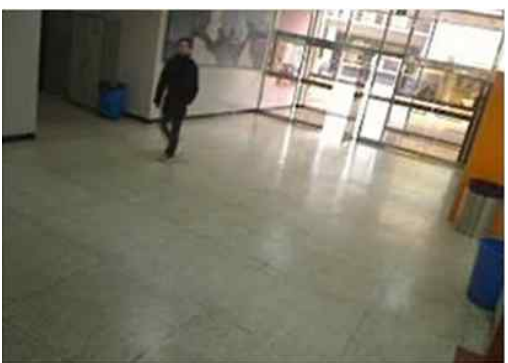

(a)

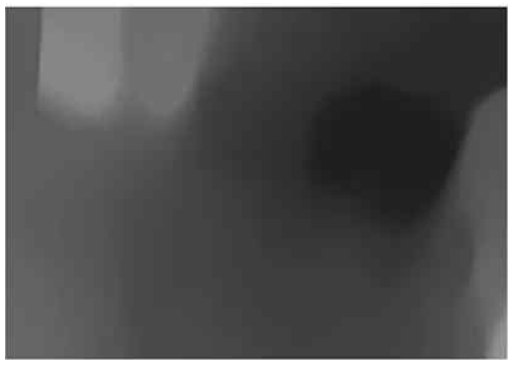

(c)

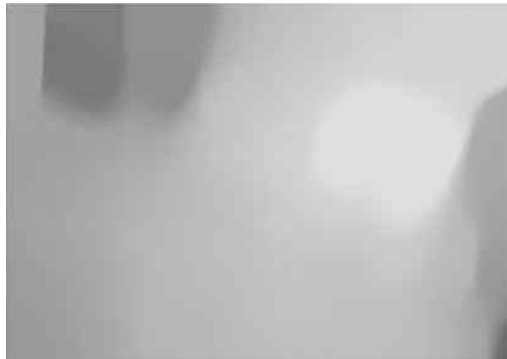

(b)

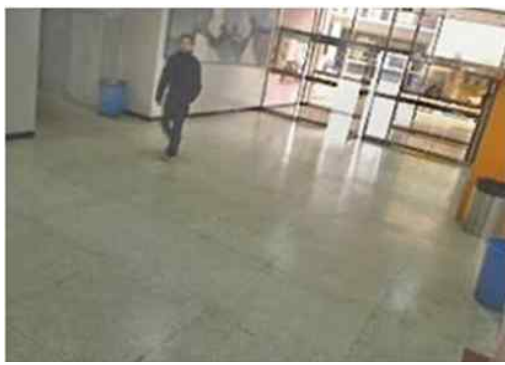

(d)

Fig. 5. An example of illumination modeling. (a) the original image (b) The illuminative component acquired by median filtering (c) The inverted image of (b) (d) result image combining (a) and (c).

removed. Using these methods a more accurate foreground area can be obtained, as shown in Fig. 6 .

2.4 Solving the occlusion problem by combining the GMM and the LKT
However, when multiple foreground objects are tracked, occlusion or overlap problems can happen In the case of exclusively using the GMM method, the occlusion problem causes the detection of imposter foreground objects. In addition, if the

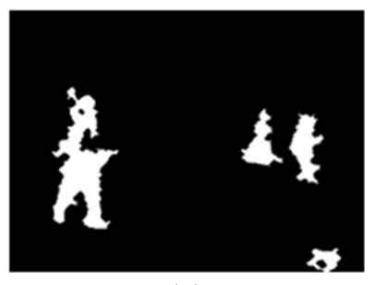

(a)

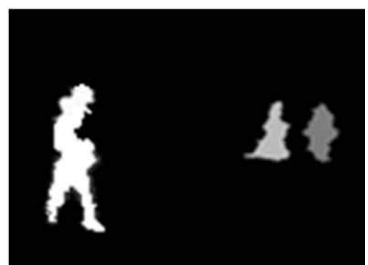

(d)

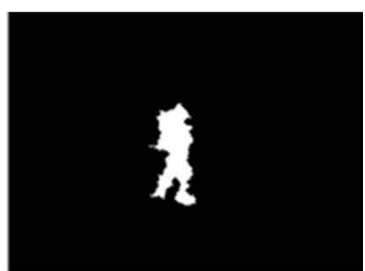

(b)

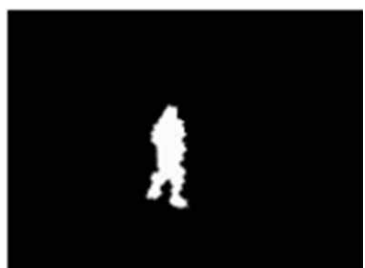

(e)

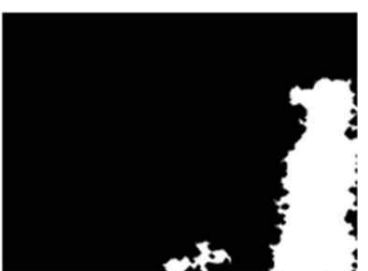

(c)

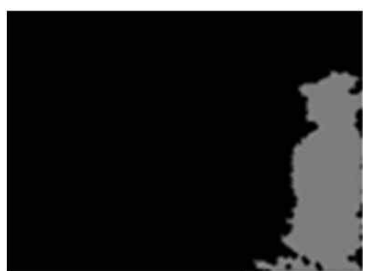

(f)

Fig. 6. The examples of the detection results by GMM after applying the illuminative normalization. (a) the result with Fig. 4 (a) (left one). (b) the result with Fig. 4 (b) (left one). (c) the result with Fig. 4 (c) (left one). In addition, the examples of the detection results obtained by further post-processing. (d) the result with Fig. 6 (a). (e) the result with Fig. 6 (b). (f) the result with Fig. 6 (c). 
tracked multiple objects are overlapped, the GMM can extract only one merged area. To overcome these problems, our new method of combining the GMM in the still image and the Lucas-KanadeTomasi (LKT) method in the successive images is proposed as follows. The LKT method calculates motion vectors, which are created based on the shifted corner points found in the successive images $[45,46]$.

The LKT method tracks feature points by calculating the correlations among them on the basis of the mean square error (MSE) [46,47]. This algorithm is represented by the following equation [11]:

$$
J(x)=I(x-d)+n(x)
$$

where $I()$ is the brightness in the current frame, $x$ is the location of the pixel, $d$ is the displacement vector, $n()$ is the noise, and $J()$ is the brightness of the next frame. As shown in Eq. (6), feature tracking is carried out in the direction that minimizes the MSE of the feature window $W$ [48].

$$
\varepsilon=\sum_{j \in W}\left(I\left(x_{j}-d\right)-J\left(x_{j}\right)\right)^{2}
$$

where $\varepsilon$ is the MSE. The brightness function needs to be approximated using the Taylor series $\mathrm{ex}^{-}$ pansion [46]. By repeating this procedure, the optimal displacement vector $d$ is found.

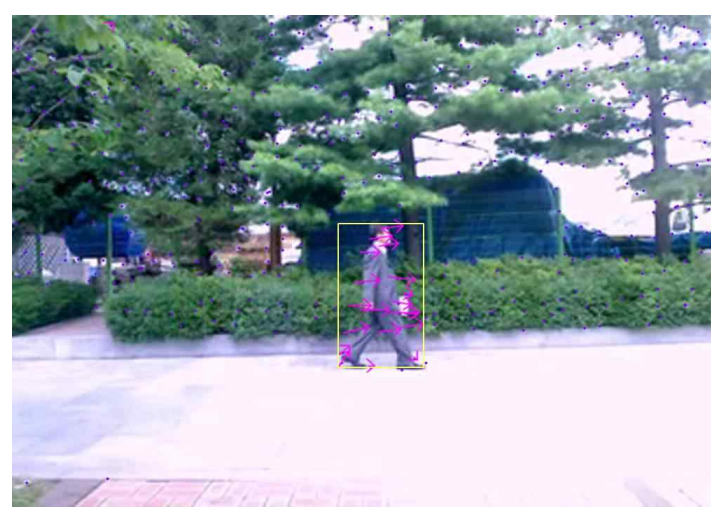

Fig. 7. The detected motion vectors by the LKT (points: the detected corner points by the LKT, arrows : the motion vectors calculated from the moved corner points in the successive images, box : the final box of human area).
Consequently, the feature points in the detected eye regions are extracted by using the Shi and Tomasi detection algorithm [49], and then, these feature points are tracked by the LKT method [48]. The detected motion vectors obtained by the LKT are shown by the arrows in Fig. 7 .

The final human area is detected in order to combine the human areas based on the GMM and the LKT. In detail, inside the box region including the detected area of GMM, the motion vectors (the arrows of Fig. 7) are located by the LKT. Based on the outmost starting and ending points of the motion vectors, one box (the box of Fig. 7) is defined as the final human area. By using these schemes, the occlusion problem can be solved between the moving objects, as shown in Fig. 8. In Fig. 8, the numbers (\#1122 \#1136) below each figure represent the frame number.

Fig. 9 shows the detection results of Fig. 3 by using proposed methods.

\subsection{The face detection using the Adaboost algorithm and face recognition based on the PCA}

Our research uses the adaptive boosting ( $\mathrm{Ada}^{-}$ boost) algorithm for detecting the face region [50, 51]. Through the training of the Adaboost algorithm, a highly accurate simple classifier is positioned in front of the hierarchical structures, which reduces false detections. Fig. 10 shows an example of the detection of the face region by using the Adaboost face detector inside of the detected human area.

Only when the width of the box detected by the GMM and the LKT is greater than the predetermined threshold is the face detection performed. In the case that it is less than the threshold, even if the face region is detected successfully, the face recognition frequently fails due to the shortage of pixel information in the facial region. The optimal threshold was empirically determined to be 50 pix ${ }^{-}$ els in order to have accurate face recognition. 


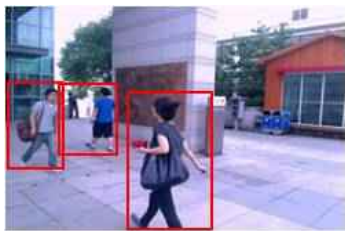

$\# 1122$

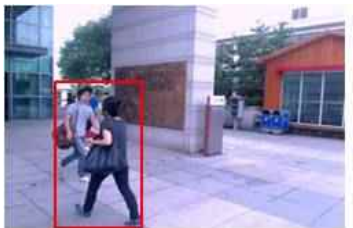

$\# 1130$

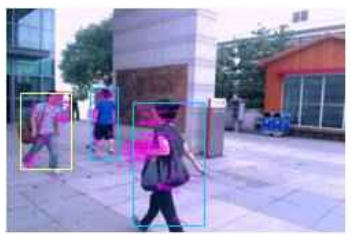

$\# 1122$

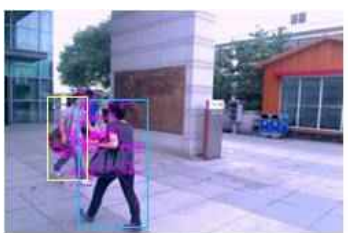

\#1130

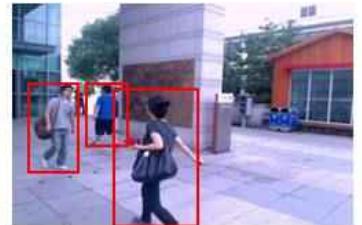

$\# 1124$

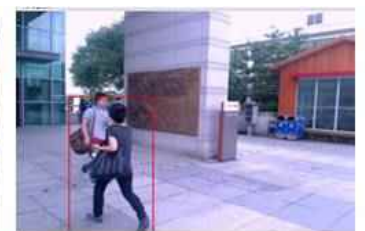

$\# 1132$

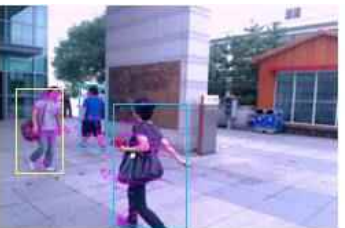

\#1124

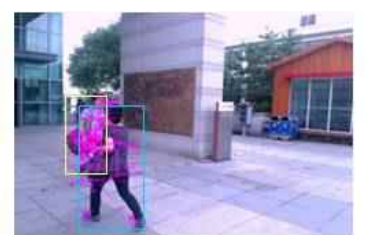

\#1132

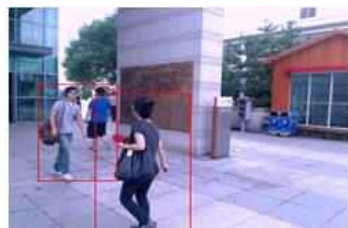

\#1126

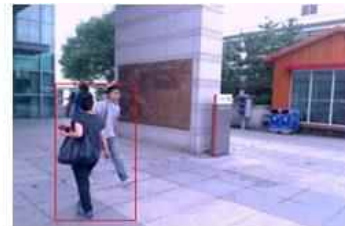

$\# 1134$

(a)

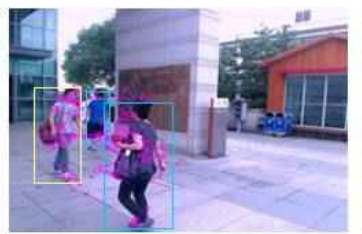

\#1126

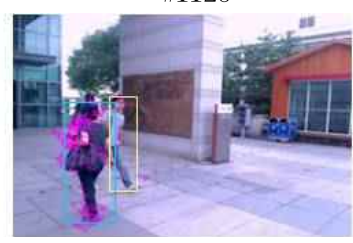

\#1134

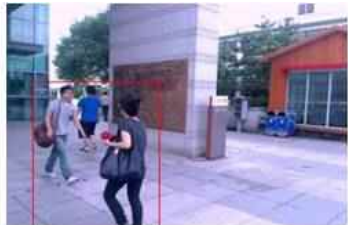

\#1128

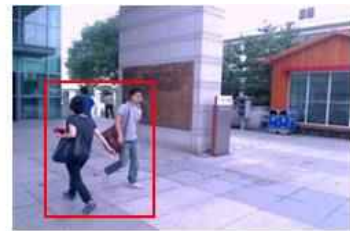

\#1136

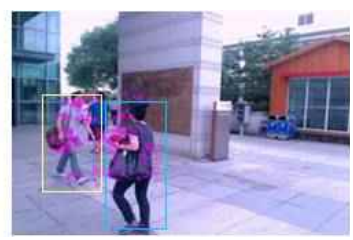

\#1128

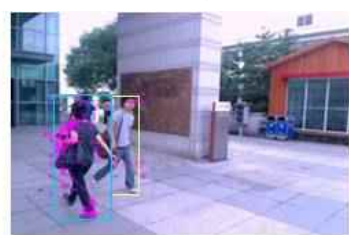

\#1136

(b)

Fig. 8. Examples of tracking multiple and overlapped objects (a) by using GMM and (b) by combining GMM and LKT.

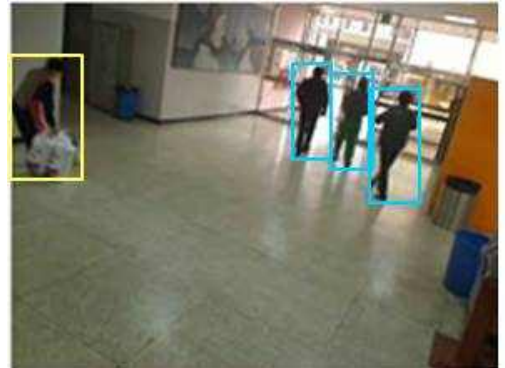

(a)

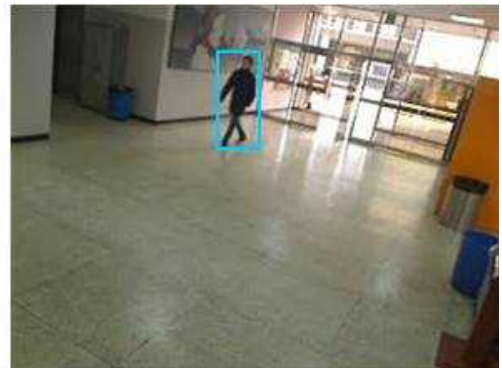

(b)

Fig. 9. The detection results of Fig. 3 by using proposed methods. (a) the case of Fig. 3 (a). (b) the case of Fig. 3 (b).

In order to reduce false face detection errors and the processing time, the face region is detected only in the predetermined area located inside the foreground region. The rough positions of the eyes and lips are detected by using binarization and component labeling in the predetermined area inside the detected face region. Based on them, a more accurate face region is defined and then is normalized 


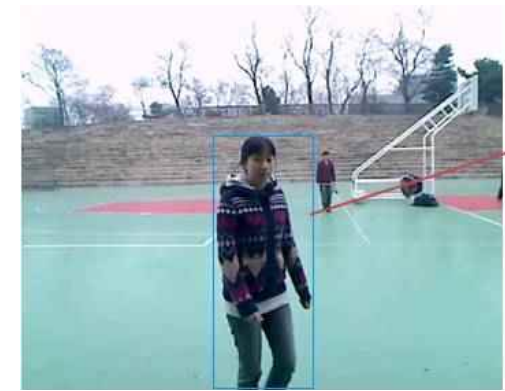

(a)

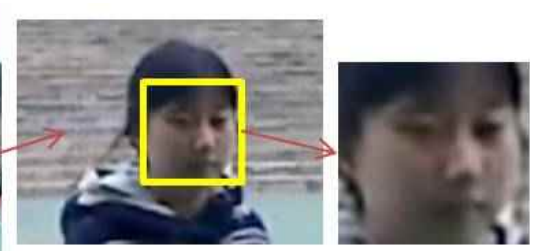

(b)

Fig. 10. An example of detecting face region by using Adaboost face detector inside of the detected human area. (a) Detected human area. (b) and (c) show the detected face region.

to $32 \times 32$ pixels for face recognition.

In this research, face recognition is performed based on the principal component analysis (PCA) method. The PCA is a statistical method which can reduce the dimensions of the original feature space, thereby an optimal feature space can be obtained [52,53]. The PCA method, being the most popular method in face recognition, is used in order to get a low dimensional component which includes the principal component from a face image. In previous research, the comparisons of PCA-based computer face recognition and human face recognition $\mathrm{ac}^{-}$ cording to facial components were also performed [54]. The obtained vectors that represent the low dimensional space are called eigen-faces, which are obtained from the training images. In general, the PCA makes eigen-vectors by using covariance matrix $C$

$$
C=\frac{1}{M} \sum_{i=1}^{M}\left(\mathbf{x}_{i}-m\right)\left(\mathbf{x}_{i}-m\right)^{T}
$$

where $\mathbf{x}_{i}$ is one dimensional vector of $i$ th face image ( $p^{\times} q$ pixels size), and $m$ is the average vector of face images of training data. By calculating covariance matrix $C$, basis vector $\mathrm{U}$ is determined by choosing $\mathrm{k}$ vectors whose magnitude of eigen-value is larger. From that, the eigen-vector $\mathrm{W}$ is determined by projecting face image $(\mathrm{x})$ to basis vector $\mathrm{U}$.

$$
\mathbf{w}=\mathbf{U}^{T}(\mathbf{x}-m)
$$

The examples of the obtained eigen-faces are shown in Fig. 11. With $\mathrm{N}$ eigen-faces, an input face image is represented as $\mathrm{N}$ eigen-coefficients. The optimal number of eigen-faces was empirically $\mathrm{de}^{-}$ termined to ensure the accuracy of the face recognition.

As shown in Fig. 10 (b), in order to match the input images having arbitrary views, the eigen-coefficients from multiple images with six different views (allowing the yaw of $-20 \sim+20 \mathrm{de}^{-}$ grees, the tilt of $-20 \sim 0$ degrees) are stored for each person in the enrollment stage (see the $\mathrm{Ex}^{-}$ perimental Results section and Fig. 14). Since the
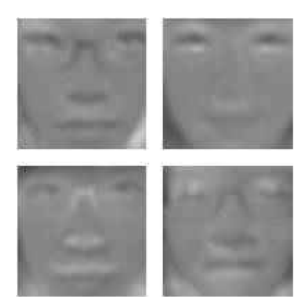
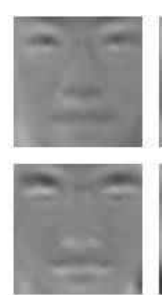
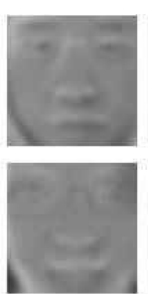
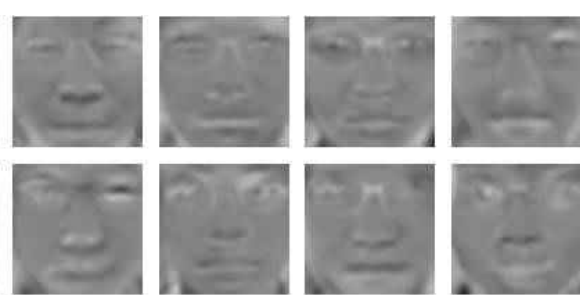

Fig. 11. The examples of the eigen-faces. 
camera is positioned above the face of human in conventional surveillance system, we do not consider the tilt greater than 0 degrees. The similarity is measured by calculating the Euclidean distance between the enrolled coefficients and those of the input image.

\section{The Experimental Results}

For the experiment, the images were collected by using a low priced and conventional webcam, such as the Logitech PN 960-000057. The spatial resolution and frame rate are $640 \times 480$ pixels and 15 frames per second, respectively [55]. A desktop computer, having an Intel® core 2TM Duo CPU $2.4 \mathrm{GHz}$ with $3 \mathrm{~GB}$ RAM, is used in experiment.

\subsection{The performance evaluation of the human tracking}

A number of image sequences were acquired according to four different scenarios and activities, as shown in Table 1 . The sequences of S1 S4 are used for testing the performance of human tracking at a distance. The sequence of $\mathrm{S} 5 \sim \mathrm{S} 8$ are used for testing the performances of both human tracking and face recognition at a closer distance. That is because the face cannot be recognized at farther distance due to the shortage of image pixel.

The performance is measured by using two measurement schemes: "Recall" and "Precision". "Recall" is the average detection rate, in which the detection is counted when the area of manually drawn is overlapped with that automatically detected. "Precision" is calculated by averaging the true positively detection rate by following equation

$$
\text { precision }=\operatorname{mean}\left(\frac{N_{t p}}{N_{t p}+N_{f p}}\right)
$$

where $N_{t p}$ is the number of true positives and $N_{f p}$ the number of false positives in the image sequence [7]. True positives mean correctly detecting object, while false positives mean incorrectly detecting objects. Consequently, if the value of Recall is close to 1 , the accuracy of people detection is high. And the value of precision is close to 1 , all the detected objects are correct with 0 false positive.

Table 2 shows the experimental result with S1

Table 1. The eight different image sequences used for the experiments

\begin{tabular}{|c|c|c|c|c|c|c|c|}
\hline $\begin{array}{l}\text { Conditions } \\
\text { Sequence } \\
\text { Index }\end{array}$ & $\begin{array}{l}\text { Indoor / } \\
\text { Outdoor }\end{array}$ & Place & Weather & $\begin{array}{l}\text { The moving } \\
\text { direction of } \\
\text { human }\end{array}$ & $\begin{array}{c}\text { The number } \\
\text { of images }\end{array}$ & $\begin{array}{l}\text { The number of } \\
\text { images in which } \\
\text { face are detected }\end{array}$ & $\begin{array}{c}\mathrm{Z} \\
\text { distance }\end{array}$ \\
\hline S1 & Indoor & Lobby & & $\begin{array}{l}\text { Omni- }^{-} \\
\text {direction }\end{array}$ & 1095 & & \multirow{4}{*}{$\begin{array}{l}\text { Farther } \\
\text { distance }\end{array}$} \\
\hline S2 & Outdoor & $\begin{array}{c}\text { Directional } \\
\text { street }\end{array}$ & Sunny & Left or right & 1335 & & \\
\hline S3 & Outdoor & Campus yard & Cloudy & Omnidirection & 1440 & & \\
\hline S4 & Outdoor & Campus yard & $\begin{array}{l}\text { Partly } \\
\text { cloudy }\end{array}$ & Omnidirection & 1680 & & \\
\hline S5 & Indoor & Front of door & & $\begin{array}{c}\text { Toward } \\
\text { (Frontal faces) }\end{array}$ & 535 & 58 & \multirow{4}{*}{$\begin{array}{c}\text { Closer } \\
\text { distance }\end{array}$} \\
\hline S6 & Indoor & Corridor & & $\begin{array}{c}\text { Omnidirection } \\
\text { (Various } \\
\text { viewing faces) }\end{array}$ & 655 & 69 & \\
\hline S7 & Outdoor & Campus yard & Cloudy & $\begin{array}{c}\text { Toward } \\
\text { (Frontal faces) }\end{array}$ & 1175 & 140 & \\
\hline S8 & Outdoor & Campus yard & Cloudy & $\begin{array}{c}\text { Omni-direction } \\
\text { (Various } \\
\text { viewing faces) }\end{array}$ & 831 & 134 & \\
\hline
\end{tabular}


Table 2. The measured "Recall" and "Precision" with the test sequence sets of S1, S2, S3, and S4 of Table 1

\begin{tabular}{|c|c|c|c|c|c|}
\hline \multirow{2}{*}{$\begin{array}{l}\text { Sets of sequences } \\
\text { Measurement }\end{array}$} & \multicolumn{2}{|c|}{ S1 } & \multirow{2}{*}{ S2 } & \multirow{2}{*}{ S4 } \\
\cline { 2 - 4 } & \multicolumn{2}{|c|}{ The included number of people in image } & & \\
\cline { 2 - 5 } & less than 3 persons & more than 3 persons & & & \\
\hline \hline Recall (\%) & 95.36 & 88.70 & 96.79 & 97.45 & 88.70 \\
\hline Precision (\%) & 97.50 & 92.13 & 93.75 & 97.94 & 92.13 \\
\hline
\end{tabular}

Table 3 The measured "Recall" and "Precision" with test sequence sets of S5, S6, S7, and S8 of Table 1

\begin{tabular}{|c|c|c|c|c|}
\hline Sets of sequences & S5 & S6 & S7 & S8 \\
\hline \hline Recall (\%) & 92.53 & 92.89 & 91.96 & 92.56 \\
\hline Precision (\%) & 90.27 & 91.4 & 91.07 & 90.85 \\
\hline
\end{tabular}

$\sim$ S4 which were collected at farther distance of Table 1 . And Table 3 shows the experimental $\mathrm{re}^{-}$ sult with S5 S8 which were collected at closer distance of Table 1.

The average recall of S1 S4 is $93.4 \%$ and average precision is $94.69 \%$. S1 is captured in the smaller place (lobby) than the others. So, if there are more than 3 persons in that place, this area is very crowded. That is the reason why only S1 is divided into two cases according to the included number of people in image. The case of more than 3 persons in S1 shows lower Recall (88.70\%) and Precision (92.13\%) compared to other cases. This is why many people are overlapped in small place. S4 shows lower Precision (92.13\%) than the others, because illumination variation frequently happens due to the influence of partly cloudy.

The average of recall of S5 - S8 is $92.49 \%$ and average of precision is $91.9 \%$. Overall Recall and Precision of $\mathrm{S} 5 \sim \mathrm{S} 8$ at closer distance sequence, are lower than those at farther distance (The average recall of $\mathrm{S} 1 \sim \mathrm{S} 4$ is $93.4 \%$, and that of $\mathrm{S} 5 \sim \mathrm{S} 8$ is $92.49 \%$. The average precision of $\mathrm{S} 1 \sim \mathrm{S} 4$ is $94.69 \%$, and that of $\mathrm{S} 5{ }^{\sim} \mathrm{S} 8$ is $91.9 \%$ ). That is because the size of object is bigger in image at closer distance, the number of background pixel is reduced, which can degrade the performance of GMM because the GMM models the background. Fig. 12 shows the examples of the tracked humans in the sets of sequences of $\mathrm{S} 1 \sim \mathrm{S} 8$.
We performed the experiments with additional open datasets, CAVIAR database [56]. These datasets include 3683 images of people walking alone, meeting with others, fighting and passing out, leaving a package in a public place, resting, and browsing, etc. Table 4 shows the experimental result with CAVIAR.

The CAVIAR datasets collected image frames with a wide angle camera lens in the entrance lobby. So, sizes of objects are relatively smaller than our datasets, and there is no illumination variation or the noise of background such as the movement of trees. Accordingly, background modeling is robust by using GMM, and precision $(99.48 \%)$ is high. But recall $(94.78 \%)$ is relatively lower than precision. It is because the sizes of objects are small, and objects are incorrectly recognized as noise, consequently. Fig. 13 shows the examples of the tracked humans by the proposed method in CAVIAR datasets.

We compared the performances of MeanShift [57,58] and CamShift tracking method [59] to those

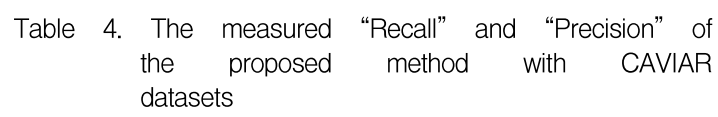

\begin{tabular}{|c|c|}
\hline $\begin{array}{l}\text { Measurement } \\
\text { Scheme }\end{array}$ & CAatabase \\
\hline \hline Recall (\%) & 94.78 \\
\hline Precision (\%) & 99.48 \\
\hline
\end{tabular}




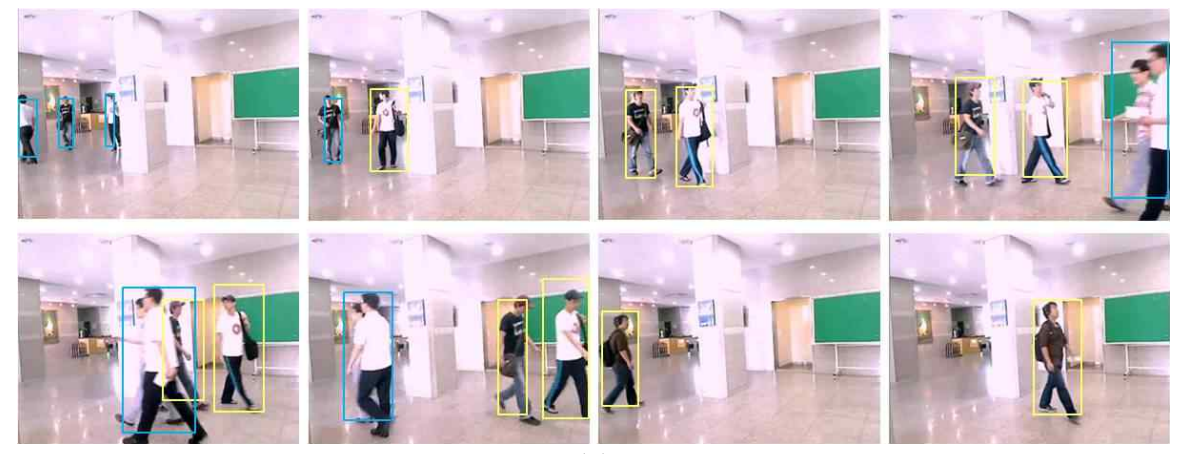

(a)

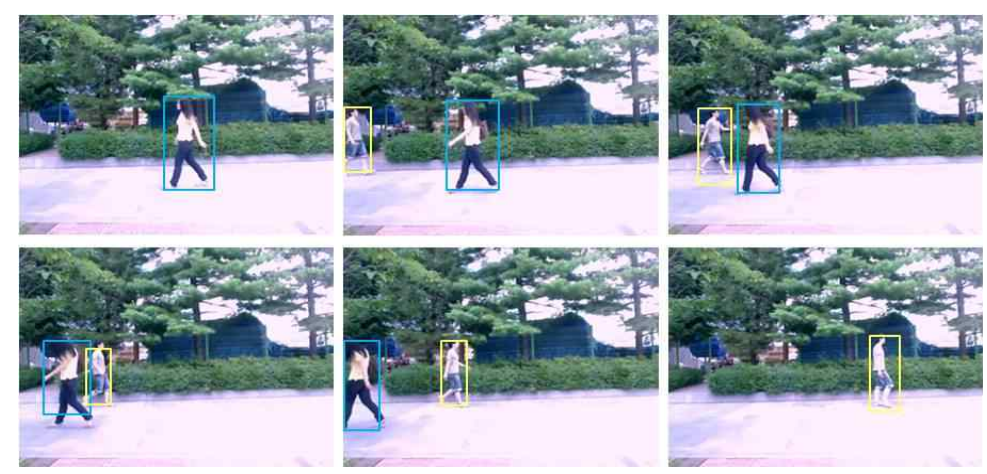

(b)
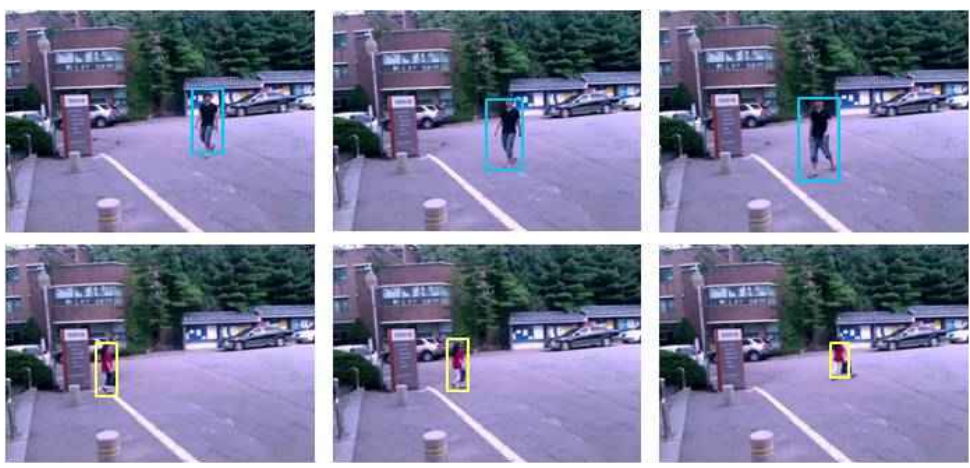

(c)
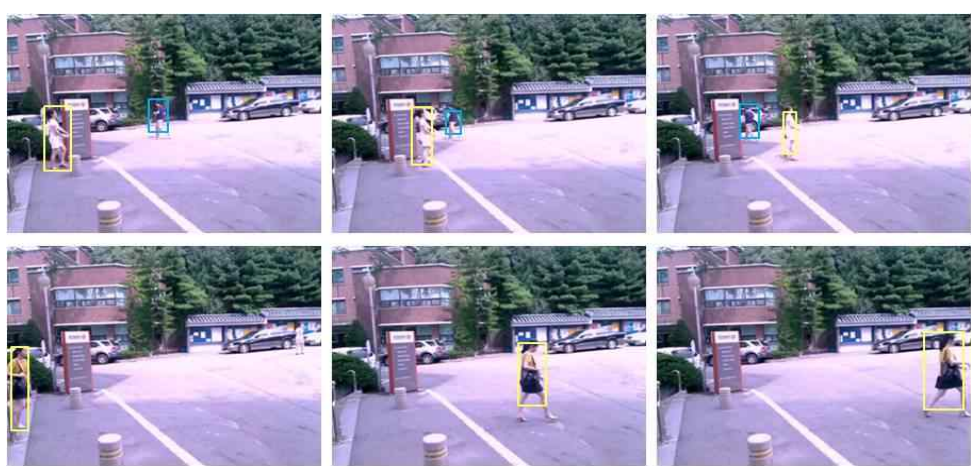

(d) 

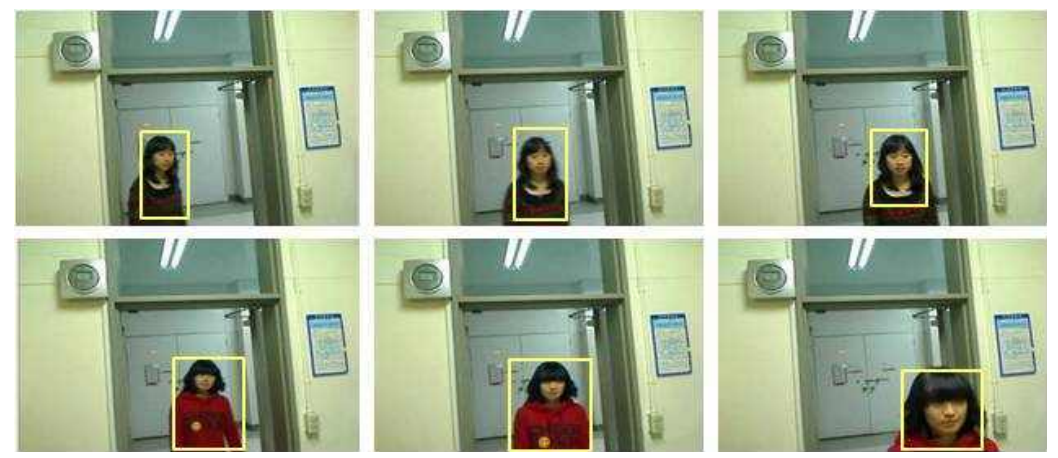

(e)
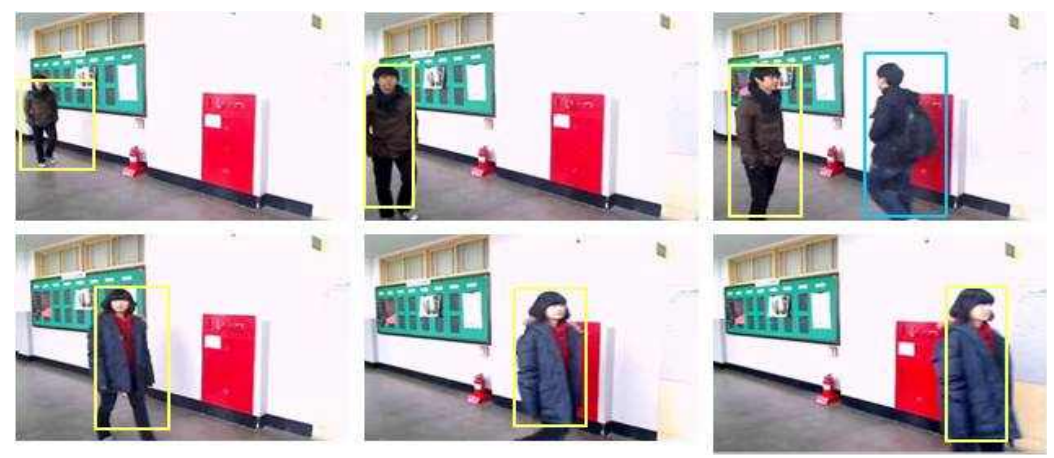

(f)
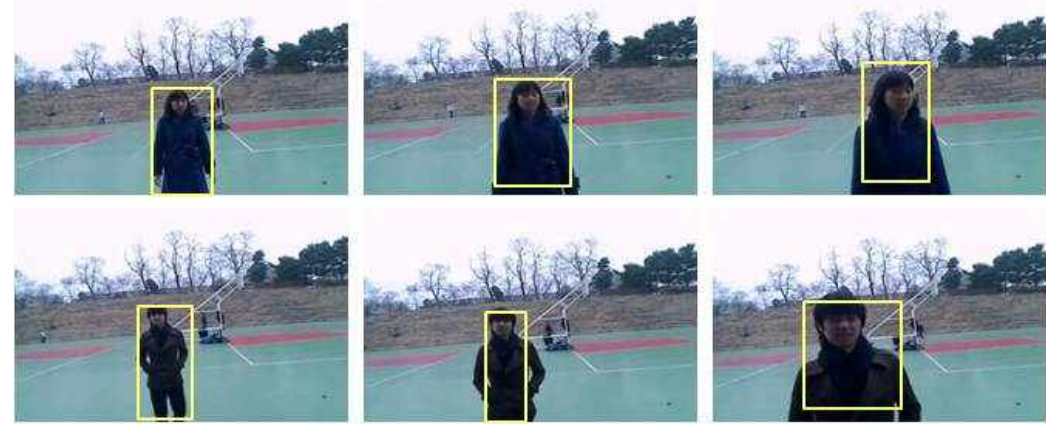

(g)
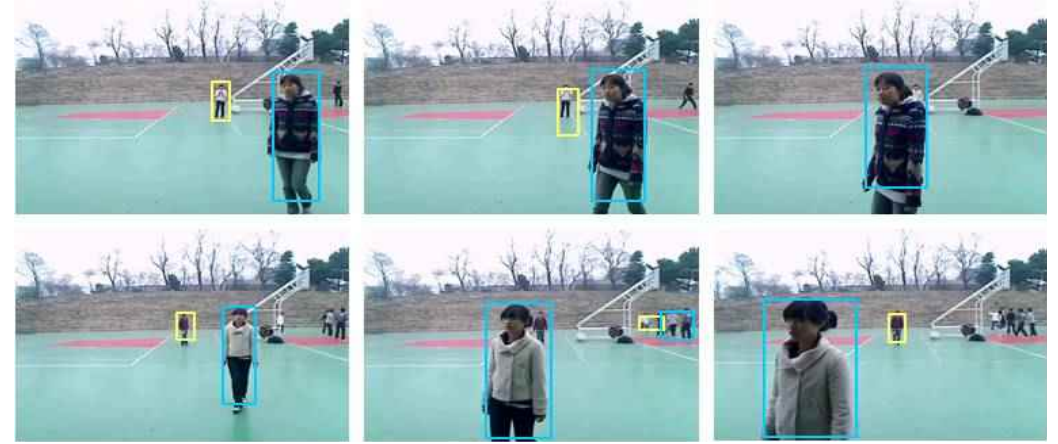

(h)

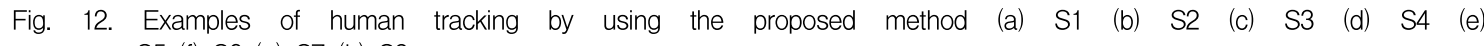
$\mathrm{S} 5$ (f) S6 (g) S7 (h) S8. 

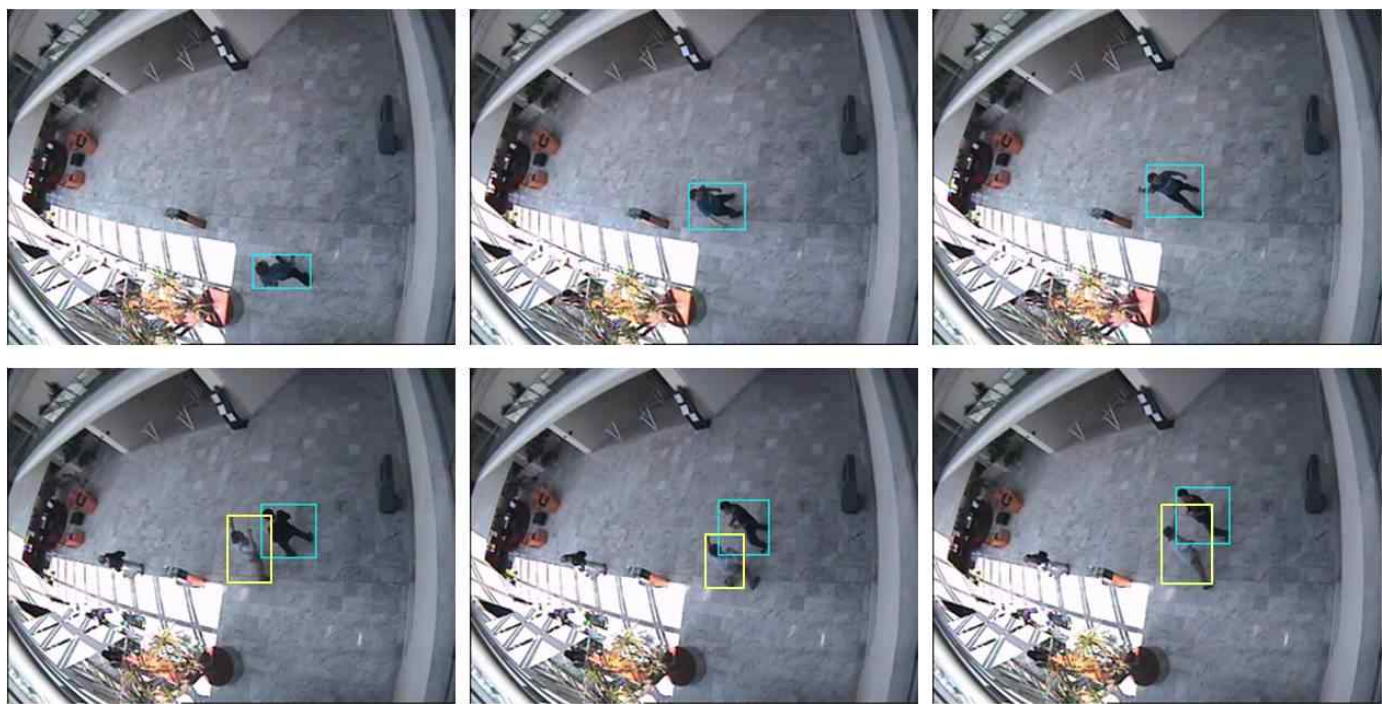

Fig. 13. the examples of the tracked humans by the propose method in CAVIAR datasets.

of the proposed method. The MeanShift and CamShift methods are based on the color histograms of the object to be tracked. In case of using the MeanShift method to track objects, the accurate kernel and parameters are required. Consequently, if the region to be tracked includes even a little background information, MeanShift method incorrectly recognizes background regions as object regions. Fig. 14 shows the examples of false detection by using MeanShift method.

To overcome these problems, CamShift can be used and we compared the proposed method to the
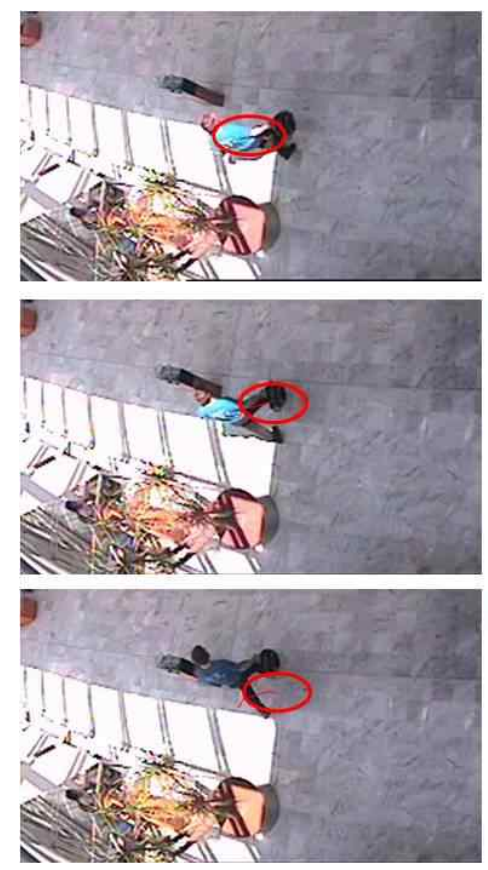
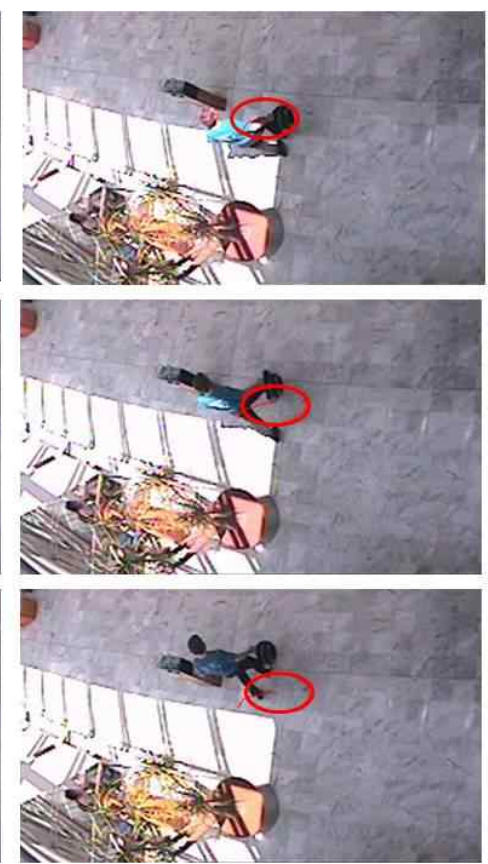
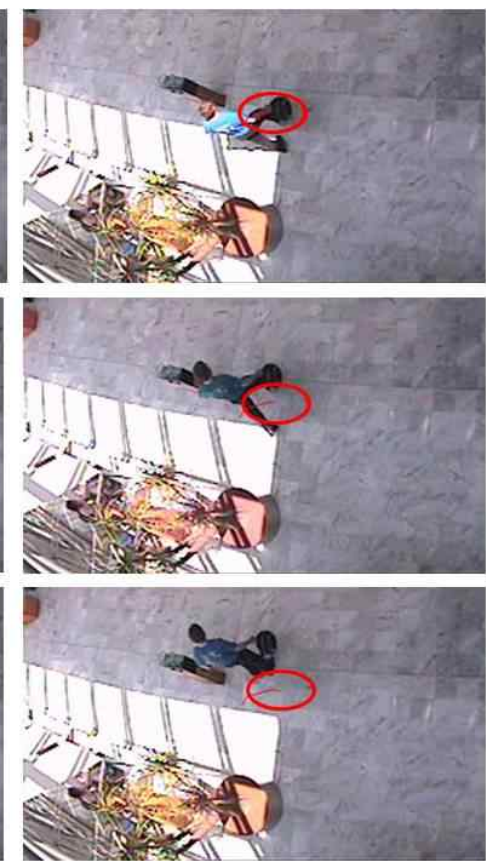

Fig. 14. the examples of false detection by using MeanShift method in CAVIAR datasets. 
Table 5. The measured "Recall" and "Precision" with our datasets and CAVIAR datasets by using CamShift method

\begin{tabular}{|c|c|c|c|c|c|c|c|c|c|}
\hline $\begin{array}{l}\text { Measurement } \\
\text { Schemes }\end{array}$ & S1 & S2 & S3 & S4 & S5 & S6 & S7 & S8 & CAVIAR \\
\hline Recall(\%) & 64.28 & 51.76 & 26.24 & 23.95 & 72.02 & 77.63 & 80.35 & 84.11 & 44.53 \\
\hline Precision(\%) & 66.93 & 49 & 35.48 & 32.1 & 78.14 & 45.32 & 74.47 & 83.45 & 53.85 \\
\hline
\end{tabular}

CamShift method. Table 5 shows the experiment results with our datasets and CAVIAR datasets by using CamShift method.

Although CamShift method has better performance than MeanShift method, it has weakness that if background is complex or the histogram of background is similar to that of objet to be tracked, tracking performances are severely decreased. The average recall of our datasets and CAVIAR is $58.32 \%$ and average precision is $57.64 \%$ by using CamShift method. As shown in Table 5, the performance of S3 (The recall is 26.24\% and precision is $35.48 \%$ ) and S4 (The recall is $23.95 \%$ and precision is $32.1 \%$ ) were worse than other cases since background regions are very complex and object regions are relatively smaller in these cases. Fig. 15 shows the examples of false tracking result with S3, S4 datasets.
S5 S8 datasets have higher accuracies (The average recall of $\mathrm{S} 5 \sim \mathrm{S} 8$ is $78.53 \%$ and average precision is $70.35 \%$ ) than $\mathrm{S} 1 \sim \mathrm{S} 4$ (The average recall of S1 $\sim \mathrm{S} 4$ is $41.56 \%$ and average precision is $45.88 \%$ ) datasets. That is because the images of $\mathrm{S} 5 \sim \mathrm{S} 8$ are collected for testing face recognition, the sizes of objects are bigger than $\mathrm{S} 1 \sim \mathrm{S} 4$ datasets. So, it is easy to track based on color histogram having many color information. But as shown in Table 5, the precision of S6 is worse than S7, S8, and S9 datasets. That is because in case of S6, there are a lot of situation that initially background is incorrectly recognized as object, and this background region continues to be recognized as object in successive images. By comparing Table $2 \sim 4$ to Table 5, we could know that the performances of the proposed method were better than previous methods with our database and open database.
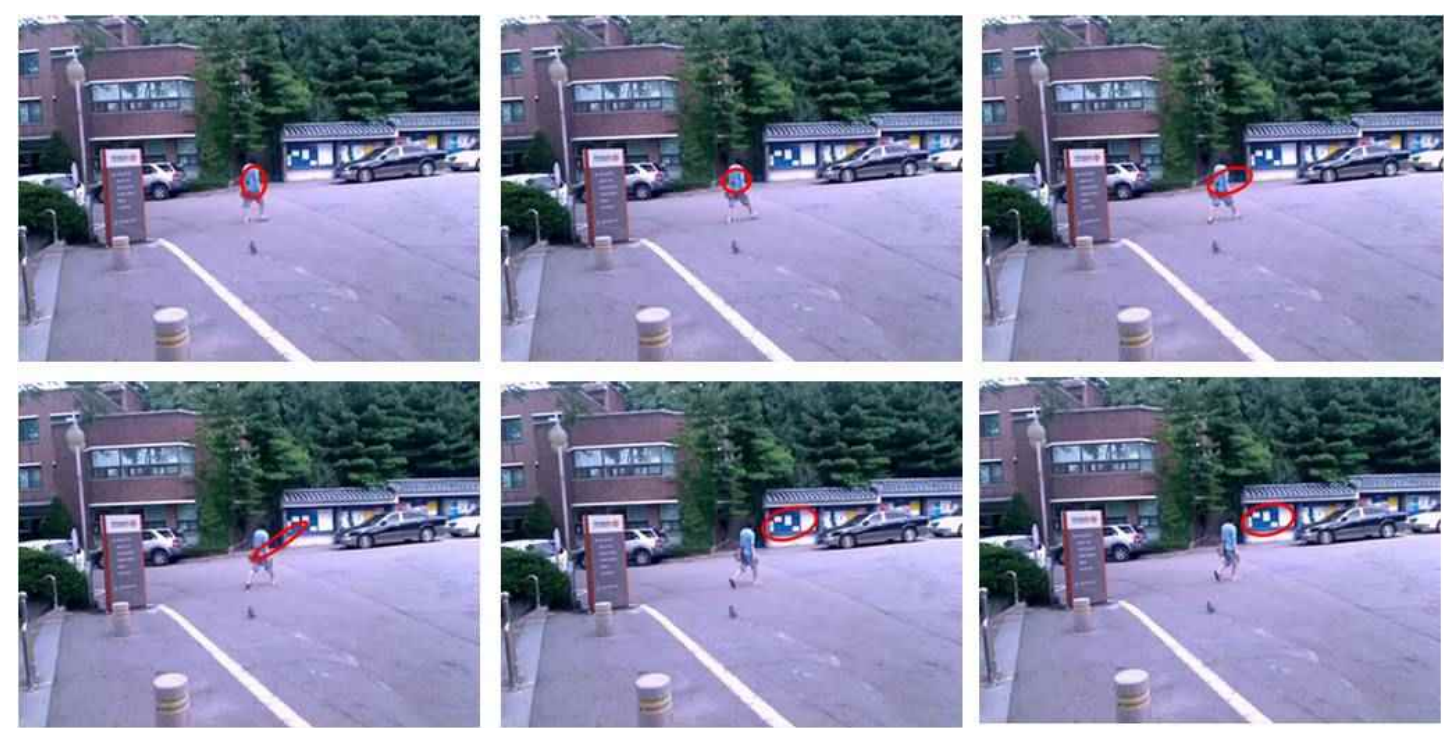

Fig. 15. the examples of false tracking result by the CamShift method with S3, S4 datasets. 


\subsection{The performance evaluation of the face recognition}

To measure the performance of face recognition, we use four sets of sequences, S5 S8, as shown in Table 1. In our experiment, open databases, such as the Notre Dame Dataset or the UCSD Dataset, were not used because they were not captured in surveillance environment including various factors, such as illumination variations, complicated background and variations of face size [60].

The eigen-coefficients from 50 persons with 6 images per person including various views are enrolled for the face recognition. Since the face image with arbitrary view can be obtained in surveillance environment as shown in Fig. 10, they include 6 directional face images: the frontal face, the left side face, the right side face, the bottom frontal face, the bottom left side face, and the bottom right side face, as shown in Fig. 16.

The images in which the viewing directions of faces are top front, top left and top right were not used for enrollment because the cameras of surveillance system are installed at high position in general

Tables 6 9 show the experimental results of 1-to-N identification. In Tables 6 9, "Rank 7"
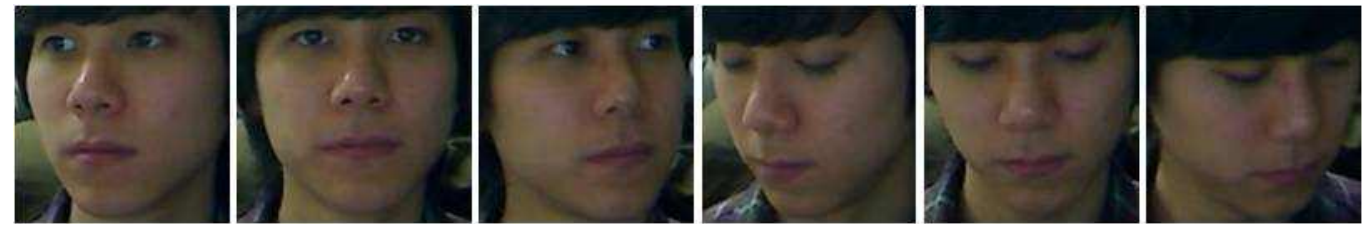

Fig. 16. 6 directional face images for enrollment

Table 6. The experimental result using S5 of Table 1

\begin{tabular}{|c|c|c|c|c|}
\hline Face detection rate (\%) & \multicolumn{4}{|c|}{ Face recognition rate (\%) } \\
\hline Frontal face & Rank 1 & $\sim$ Rank 3 & $\sim$ Rank 5 & $\sim$ Rank 7 \\
\hline \hline 93.1 & 74.07 & 94.44 & 100 & 100 \\
\hline
\end{tabular}

Table 7. The experimental result using S6 of Table 1

\begin{tabular}{|c|c|c|c|c|c|}
\hline \multicolumn{2}{|c|}{ Face detection rate (\%) } & \multicolumn{4}{|c|}{ Face recognition rate (\%) } \\
\cline { 1 - 2 } Frontal face & Side face & Rank 1 & $\sim$ Rank 3 & $\sim$ Rank 5 & $\sim$ Rank 7 \\
\hline \hline 94.74 & 54.00 & \multirow{2}{*}{57.78} & 68.89 & 84.44 & 100 \\
\hline \multicolumn{2}{|r|}{65.22} & & & \multirow{2}{*}{5} & \\
\hline
\end{tabular}

Table 8. The experimental result using S7 of Table 1

\begin{tabular}{|c|c|c|c|c|}
\hline Face detection rate (\%) & \multicolumn{4}{|c|}{ Face recognition rate (\%) } \\
\hline Frontal face & Rank 1 & $\sim$ Rank 3 & $\sim$ Rank 5 & $\sim$ Rank 7 \\
\hline 90 & 82.54 & 95.24 & 100 & 100 \\
\hline
\end{tabular}

Table 9. The experimental result using S8 of Table 1

\begin{tabular}{|c|c|c|c|c|c|}
\hline \multicolumn{2}{|c|}{ Face detection rate $(\%)$} & \multicolumn{4}{|c|}{ Face recognition rate $(\%)$} \\
\hline Frontal face & Side face & Rank 1 & $\sim$ Rank 3 & $\sim$ Rank 5 & $\sim$ Rank 7 \\
\hline 92 & 50.46 & \multirow{2}{*}{73.08} & \multirow{2}{*}{91.03} & \multirow{2}{*}{100} & \multirow{2}{*}{100} \\
\hline & & & & & \\
\hline
\end{tabular}


represents that the accuracy of the face recognition through counting results in the 1 st $\sim 7$ th candidates matching. That is, if the corrected matching face is same to or higher rank than the 7th of the whole matching list (e.g. 1st, 2nd $\cdots 7$ th rank), it is $\mathrm{re}^{-}$ garded as a correct recognition.

The average rank 1 rate of face recognitions of Table $6 \sim 9$ is $71.87 \%$, the average rank 3 rate is $87.4 \%$, the average rank 5 rate is $96.11 \%$, and the average rank 7 rate is $100 \%$. The experimental results showed that the matching results of the face images captured in the outdoor environment were better than those of the indoor environment (The rank 1 rate $(82.54 \%)$ of face recognition of $\mathrm{S} 7$ is higher than that $(74.07 \%)$ of S5, And the rank 1 rate $(73.08 \%)$ of face recognition of $\mathrm{S} 8$ is higher than that (57.78\%) of S6). This is the reason why we performed the illuminative normalization of the $32 \times 32$ face image. In most cases, the result in outdoor environment showed a better performance because the natural environmental light is more uniform than that in the indoor environment. Also, the image sequence including only frontal faces showed better performance than those having various viewing faces. So, S7 shows the best performance (The rank 1 rate of face recognition is 82.54\%), while S6 shows the worst performance (The rank 1 rate of face recognition is 57.78\%). In all cases, the face recognition rate becomes 100\% in Rank 7. So, we can know that the proposed method can be used in the surveillance monitoring system with human administrator, which is often the case with conventional intelligent surveillance system. Fig. 17 shows the examples of correctly matching as "Rank 1". Fig. 18 shows example of incorrectly matching.

Experimental results showed that the total proc ${ }^{-}$ essing time is $90.6 \mathrm{~ms}$ (11 frames/sec).

$\mathrm{S} 1 \sim \mathrm{S} 4$ datasets were captured without any control, supervision and cooperate of image providers. But S5 S8 datasets were captured under control and supervision in order to measure especially the performance of face recognition. That is because if the sizes of faces are too small or directions of faces are too rotated, the face recognition cannot be done. However, considering the actual surveillance environment, S5 S8 datasets were collected with the minimal instruction of moving direction and distance. By conclusion, S1 S4 and additional open database of CAVIAR were mainly used for measuring the performance of tracking. And S5 S8 were mainly used for the performances of tracking and face recognition.

\section{CONCLUSIONS}

In this paper, a new surveillance system that is robust for illumination variation and which can

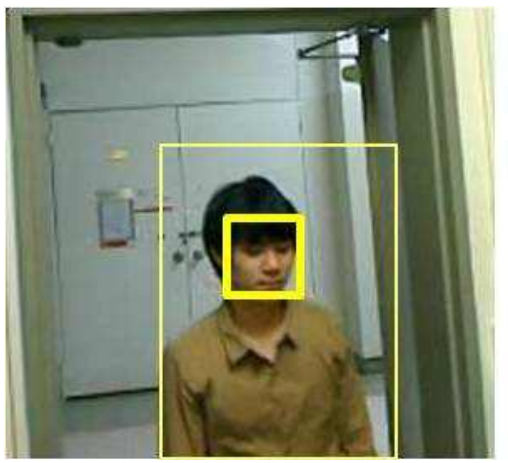

(a)

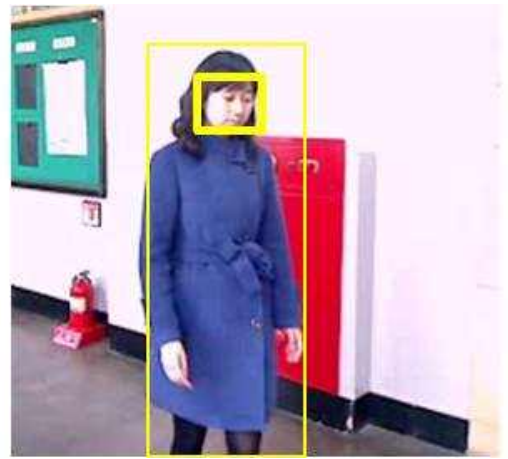

(b)

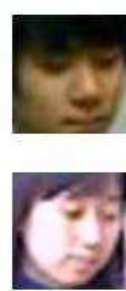

(c)

Fig. 17. Example of correctly matching in face recognition system (a) original image (b) detected face image (c) the face images for enrollment which are correctly matched. 


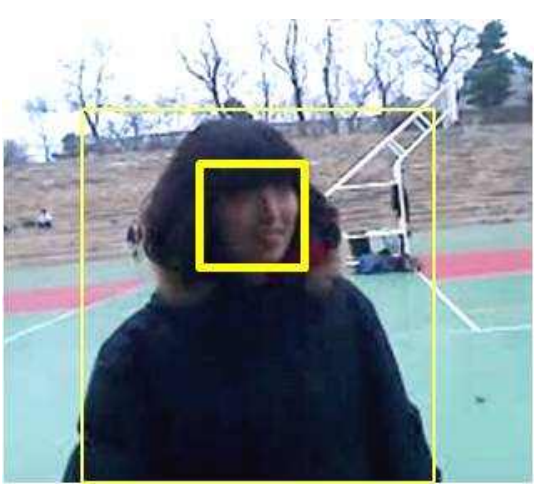

(a)

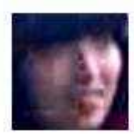

(b)

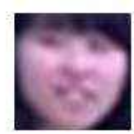

(c)

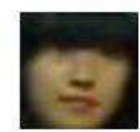

(d)

18. Example of incorrectly matching in face recognition system (a) original image (b) detected face image (c) Rank 1 matching image (d) the face image for enrollment of detected person in (a).

track multiple people irrespective of occlusion and overlap is proposed. The proposed research has following novelties.

(1) The illuminative variations and shadow regions are reduced by an illumination normalization based on the median and inverse filtering of the $\mathrm{L} * \mathrm{a} * \mathrm{~b} *$ image.

(2) The multiple occluded and overlapped people are tracked by combining the GMM in the still image and the Lucas-Kanade-Tomasi (LKT) method in successive images.

(3) With the proposed human tracking and the existing face detection \& recognition methods, the tracked multiple people are successfully identified.

The experimental results show that the average recall of our datasets and CAVIAR is $93.17 \%$ and average precision is $93.65 \%$. The average of rank 1 rate of face recognition is $71.87 \%$, the rank 3 rate is $87.4 \%$, the rank 5 rate is $96.11 \%$, and the rank 7 rate is $100 \%$, respectively.

In future work, we would test the proposed method in more varied environments. We also plan to study a method for increasing the performance of face detection and face recognition irrespective of the viewing directions of the face.

\section{REFERENCES}

[1] W. Hu, T. Tan, L. Wang, and S. Maybank,
"A Survey on Visual Surveillance of Object Motion and Behaviors," IEEE Trans. on Sys ${ }^{-}$ tems, Man, and Cybernetics, Part C: Applications and Reviews, Vol.34, No.3, pp. 334-352, 2004

[2] A. Dore, M. Soto, and C.S. Regazzoni, "Bayesian Tracking for Video Analytics," IEEE Signal Processing Magazine, Vol.27, Issue 5, pp. 46-55, 2010.

[3] V. Saligrama, J. Konrad, and P. Jodoin, "Video Anomaly Identification," IEEE Signal Processing Magazine, Vol.27, Issue 5, pp. 18-33, 2010.

[4] J. Houser and L. Zong, "The ARL Multimodal Sensor: A Research Tool for Target Signature Collection, Algorithm Validation, and Emplacement Studies," Proc. International Conference on Computer Vision and Pattern Recognition, 2007. (페이지 정보가 없습니다)

[5] Z. Zhu, W. Li, E. Molina, and G. Wolberg, "LDV Sensing and Processing for Remote Hearing in a Multimodal Surveillance System," Proc. International Conference on Computer Vision and Pattern Recognition, 2007. (페이지 정보가 없습니다)

[6] P. Smaragdis, B. Raj, and K. Kalgaonkar, "Sensor and Data Systems, Audio-Assisted Camera, and Acoustic Doppler Sensors," Proc. International Conference on Computer 
Vision and Pattern Recognition, 2007. (페이지 정보가 없습니다)

[7] C. Town, "Sensor Fusion and Environmental Modeling for Multimodal Sentient Computing," Proc. International Conference on Computer Vision and Pattern Recognition, 2007. (페이지 정보가 없습니다)

[8] T. Zhao, M. Aggarwal, T. Germano, L. Roth, A. Knowles, R. Kumar, H. Sawhney, and S. Samaraskera, "Toward a Sentient Environment: Real-time Wide Area Multiple Human Tracking with Identities," Machine Vision and Applications, Vol.19, No.5-6, pp. 301-314, 2008.

[ 9 ] I. Haritaoglu, D. Harwood, and L.S. Davis, "W4 : Real-time Surveillance of People and Their Activities," IEEE Trans. on Pattern Analysis Machine Intelligence, Vol.22, Issue 8, pp. 809- 830, 2000.

[10] B. Wu and R. Nevatia, "Tracking of Multiple, Partially Occluded Humans Based on Static Body Part Detection," Proc. International Conference on Computer Vision and Pattern Recognition, Vol.1, pp. 951-958, 2006.

[11] H. Lim, V.I. Morariu, O.I. Camps, and M. Sznaier, "Dynamic Appearance Modeling for Human Tracking," Proc. International Conference on Computer Vision and Pattern Recognition, Vol.1, pp. 751-757, 2006.

[12] T. Zhao, R. Nevatia, and F. Lv, "Segmentation and Tracking of Multiple Humans in Complex Situations," IEEE Trans. on Pattern Analysis and Machine Intelligence, Vol.26, No.9, pp. 1208-1221, 2004.

[13] Y. Wum and T. Yu, "A Field Model for Human Detection and Tracking," IEEE Trans. on Pattern Analysis and Machine Intelligence, Vol.28, No.5, pp. 753-765, 2006.

[14] J. Varona, J. Gonzàlez, I.R. Juan, and J. Villanueva, "Importance of Detection for Video Surveillance Applications," Optical Engineering, Vol.47, No.8, pp. 087201-1-087201-9,
2008.

[15] W. Lin, M.T. Sun, R. Poovendran, and Z. Zhang, "Activity Recognition using a Combination of Category Components and Local Models for Video Surveillance," IEEE Trans. on Circuits and Systems for Video Technology, Vol.18, No.8, pp. 1128-1139, 2008.

[16] T. Xiang and S. Gong, "Activity Based Surveillance Video Content Modeling," Pattern Recognition, Vol.41, Issue 7, pp. 2309-2326, 2008.

[17] D. Makris, T. Ellis, and J. Black, "Intelligent Visual Surveillance: Towards Cognitive Vision Systems," The Open Cybernetics and Systemics Journal, Vol.2, No.??, pp. 219-229, 2008. (호 기입 요함!!!) (본 저널의 형식상 호가 없습 니다.)

[18] Z. Liu, K. Huang, T. Tan, and L. Wang, "Cast Shadow Removal with GMM for Surface $\mathrm{Re}^{-}$ flectance Component," Proc. International Conference on Pattern Recognition, Vol.1, pp. 727-730, 2006.

[19] Y. Matsushita, K. Nishino, K. Ikeuchi, and M. Sakauchi, "Shadow Elimination for Robust Video Surveillance," Proc. Workshop on Motion and Video Computing, pp. 15-21, 2002.

[20] R. Liu, X. Gao, R. Chu, X. Zhu, and S. Z. Li, "Tracking and Recognition of Multiple Faces at Distances," Advances in Biometrics, Vol.4642, pp. 513-522, 2007.

[21] L. Jiangwei and W. Yunhong, "Video-Based Face Tracking and Recognition on Updating Twin GMMs," Advances in Biometrics, Vol.4642, pp. 848-857, 2007.

[22] N. Friedman and S. Russell, "Image Segmentation in Video Sequences: a Probabilistic Approach," Proc. the 13th Conf. Uncertainty in Artificial Intelligence, pp. 1-3, 1997.

[23] D. Koller, J. Weber, T. Huang, J. Malik, G. Ogasawara, B. Rao, and S. Russel, "Toward Robust Automatic Traffic Scene Analysis in Real-time," Proc. International Conference 
on Pattern Recognition, pp. 126-131, 1994.

[24] M. Köhle, D. Merkl, and J. Kastner, "Clinical Gait Analysis by Neural Networks: Issues and Experiences," Proc. IEEE Symposium on Computer-Based Medical Systems, pp. 138143, 1997.

[25] H. Z. Sun, T. Feng, and T. N. Tan, "Robust Extraction of Moving Objects from Image Sequences," Proc. Asian Conf. Computer Vision, pp. 961-964, 2000.

[26] W.E.L. Grimson, C. Stauffer, R. Romano, and L. Lee, "Using Adaptive Tracking to Classify and Monitor Activities in a Site," Proc. IEEE Conference on Computer Vision and Pattern Recognition, pp. 22-31, 1998.

[27] S. McKenna, S. Jabri, Z. Duric, A. Rosenfeld, and H. Wechsler, "Tracking Groups of People," Computer Vision Image Understanding, Vol.80, No.1, pp. 42-56, 2000

[28] A.J. Lipton, H. Fujiyoshi, and R.S. Patil, "Moving Target Classification and Tracking from Real-time Video," Proc. IEEE Workshop Applications of Computer Vision, pp. 814, 1998

[29] D. Meyer, J. Denzler, and H. Niemann, "Model Based Extraction of Articulated Objects in Image Sequences for Gait Analysis," Proc. IEEE International Conference on Image Processing, pp. 78-81, 1998.

[30] J. Barron, D. Fleet, and S. Beauchemin, "Performance of Optical Flow Techniques," International Journal of Computer Vision, Vol.12, No.1, pp. 42-77, 1994.

[31] D. Meyer, J. Psl, and H. Niemann, "Gait Classification with HMM's for Trajectories of Body Parts Extracted by Mixture Densities," Proc. British Machine Vision Conference, pp. 459-468, 1998.

[32] C. Stauffer and W.E.L. Grimson, "Adaptive Background Mixture Models for Real-time Tracking," Proc. International Conference on Computer Vision and Pattern Recognition,
Vol.2, pp. 246-252, 1999.

[33] Y. Tian, M. Lu, and A. Hampapur, "Robust and Efficient Foreground Analysis for Realtime Video Surveillance," Proc. International Conference on Computer Vision and Pattern Recognition, Vol.1, pp. 1182-1187, 2005.

[34] G.P. Nam, B.J. Kang, and K.R. Park, "Robustness of Face Recognition to Variations of Illumination on Mobile Devices Based on SVM," KSII Transactions on Internet and Information Systems, Vol.4, No.1, pp. 25-44, 2010.

[35] G.P. Nam, B.J. Kang, E.C. Lee, and K.R. Park, "New Fuzzy-based Retinex Method for the Illumination Normalization of Face Recognition on Mobile Device," IEEE Transactions on Consumer Electronics, in submission.

[36] D. Swets and J. Weng, "Discriminant Analysis and Eigenspace Partition Tree for Face and Object Recognition from Views," Proc. International Conference on Automatic Face and Gesture Recognition, pp. 182-187, 1996.

[37] B. Moghaddam, W.Wahid, and A. Pentland, "Beyond Eigenfaces: Probabilistic Matching for Face Recognition," Proc. International Conference on Automatic Face and Gesture Recognition, pp. 30-35, 1998.

[38] G. Guo, S. Li, and K. Chan, "Face Recognition by Support Vector Machines," Proc. International Conference on Automatic Face and Gesture Recognition, pp. 196-201, 2000.

[39] H. Rowley, S. Baluja, and T. Kanade, "Neural Network Based Face Detection," IEEE Trans. on Pattern Analysis and Machine Intelligence, Vol.20, Issue 1, pp. 23-38, 1998.

[40] B. Menser and M. Wien, "Segmentation and Tracking of Facial Regions in Color Image Sequences," Proc. SPIE Visual Communications and Image Processing, Vol.4067, pp 731-740, 2000

[41] A. Saber and A.M. Tekalp, "Frontal-view Face Detection and Facial Feature Extraction 
using Color, Shape and Symmetry Based Cost Functions," Pattern Recognition, Vol.19, No.8, pp. 669-680, 1998.

[42] P. KaewTraKulPong, and R. Bowden, "An Improved Adaptive Background Mixture Model for Real-time Tracking with Shadow Detection," Proc. the 2nd European Workshop on Advanced Video Based Surveillance Systems, pp. 1-5, 2001.

[43] G.D. Hines, Z. Rahman, D.J. Jobson, and G.A. Woodell, "Single-scale Retinex Using Digital Signal Processors," Proc. Global Signal Processing Expo, pp. 335-343, 2004.

[44] R. Cucchiara, C. Grana, M. Piccardi, and A. Prati, "Detecting Moving Objects, Ghosts, and Shadows in Video Streams," IEEE Trans. on Pattern Analysis and Machine Intelligence, Vol.25, No.10, pp. 1337-1342, 2003.

[45] B.D. Lucas and T. Kanade, "An Iterative Image Registration Technique with an Application to Stereo Vision," Proc. International Joint Conference on Artificial Intelligence, pp. 674-679, 1981.

[46] C. Tomasi and T. Kanade, Detection and Tracking of Point Features, Technical Report CMU-CS-91-132, Carnegie Mellon University, 1991.

[47] J.Y. Bouguet, Pyramidal Implementation of the Lucas Kanade Feature Tracker Description of the Algorithm, Intel Corporation, Microprocessor Research Labs. OpenCV Documents, 2003.

[48] W.O. Lee, E.C. Lee, and K.R. Park, "Blink Detection Robust to Various Facial Poses," Journal of Neuroscience Method, Vol.193, No.2, pp. 356-372, 2010.

[49] J. Shi, C. Tomasi, "Good Features to Track," Proc. Int Conference on CVPR, pp. 593-600, 1994.

[50] Y. Freund and R.E. Schapire, "A Short
Introduction to Boosting," Journal of Japanese Society for Artificial Intelligence, Vol.14, No.5, pp. 771-780, 1999

[51] P. Viola and M.J. Jones, "Robust Real-Time Face Detection," International Journal of Computer Vision, Vol.57, No.2, pp. 137-154, 2004.

[52] H. Abdi and L.J. Williams, "Principal Component Analysis," Computational Statistics, Vol 2, issue 4, pp. 433-459, 2010

[53] M. Turk and A. Pentland, "Eigenfaces for Recognition," Journal of Cognitive Neuroscience, Vol.3, No.1, pp. 71-86, 1991.

[54] H.H. Nam, B.J. Kang, and K.R. Park, "Comparison of Computer and Human Face Recognition According to Facial Components," Journal of Korea Multimedia Society, Vol.15, No.1, pp. 40-50, 2012

[55] Logitech Camera, http://www.logitech.com (accessed on December 24, 2011)

[56] http://homepages.inf.ed.ac.uk/rbf/CAVIAR/ (accessed on December 24, 2011)

[57] D. Comaniciu, V. Ramesh, and P. Meer, "Kernel-Based Object Tracking", IEEE Trans. on Pattern Analysis and Machine Intelligence, Vol.25, No.5, pp. 564-577, 2003.

[58] D. Comaniciu, V. Ramesh, and P. Meer, "RealTime Tracking of Non-Rigid Objects using Mean Shift," Proc. IEEE Conference on Computer Vision and Pattern Recognition (CVPR OO), Vol.2, pp. 142-149, 2000.

[59] G.R. Bradski, Computer Video Face Tracking for Use in a Perceptual User Interface, Technical report, Intel Technology Journal, Q2, 1998.

[60] D. Thomas, K.W. Bowyer, and P.J. Flynn "Strategies for Improving Face Recognition from Video," Advances in Biometrics, pp. 339-361, 2008. 


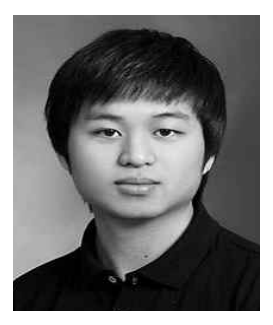

\section{Won Oh Lee}

2009. 2. Bachelor in Dept. of Electronics Engineering, Dongguk University

Current: Unified Master and Doctorial in Div. of Electronics Electrical Engineering, Dongguk University

Research interests : biometrics, image processing, pattern recognition

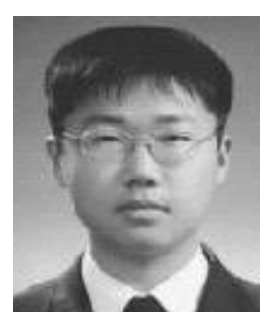

\section{Young Ho Park}

2010.2. Bachelor in Dept. of Electronics Engineering, Dongguk University

$\sim$ Current: Master in Div. of Electronics Electrical Engineering, Dongguk University Research interests : biometrics, image processing

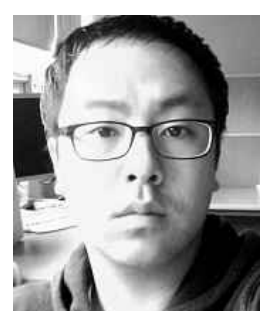

\section{Eui Chul Lee}

- 2005.2. 7 7Bachelor in Dept. of software, Sangmyung University

- 2007.2. Master in Dept. of Computer Science, Sangmyung University

- 2010.2. Ph.D in Dept. of Computer Science, Sangmyung University

$\sim 2012$.2. Senior researcher in NIMS

$\sim$ Current: Full time lecture in Dept. of Computer Science, Sangmyung University Research interests: computer vision, image processing, pattern recognition, egonomics, $\mathrm{BCI}$ and $\mathrm{HCI}$

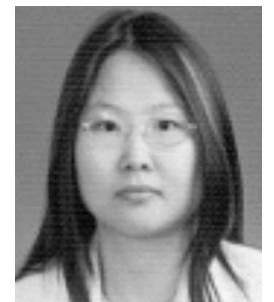

Heekyung Lee

1999. 2. Bachelor in Dept. of Computer Eng., Yeungnam University

- 2002. 2. Master in Dept. of Engineering, Information and Communication University (ICU)

$\sim$ Current: Senior engineering staff in Electronics and Telecommunications Research Institute (ETRI)

Research interests : personalized service via metadata, $\mathrm{HCI}$, bi directional $\mathrm{AD}$, and video content analysis.

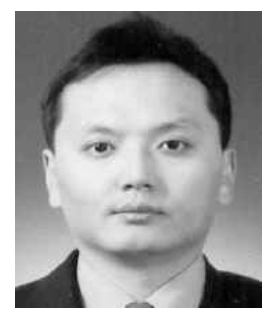

\section{Kang Ryoung Park}

1994. 2. Bachelor in Dept. of Electronics Eng., Yonsei University

1996. 2. Master in Dept. of Electronics Eng., Yonsei University

2000. 2. Ph.D in Dept. of Electrical and Computer Eng., Yonsei University

$\sim$ 2003. 2. Senior researcher in LG Elite

$\sim 2008$. 2. Full time lecturer and assistant professor in Div. of Digital Media Tech., Sangmyung University.

Current: Assistant professor and associate professor in Div. of Electronics and Electrical Eng., Dongguk University

Research interests: image signal processing, pattern recognition, biometrics 